\title{
Uncovering Flexible Active Site Conformations of SARS-CoV-2 3CL Proteases through Protease Pharmacophore Clusters and COVID-19 Drug Repurposing
}

Nikhil Pathak ${ }^{1,2,3}$, Yun-Ti Chen ${ }^{3}$, Yen-Chao Hsu ${ }^{3}$, Nung-Yu Hsu ${ }^{3}$, Chih-Jung Kuo ${ }^{4}$, Hui Ping Tsai ${ }^{5}$, Jaw-Jou Kang ${ }^{6}$, ChihHeng Huang ${ }^{5,7}$, Sui-Yuan Chang ${ }^{8,9}$, Yu-Hsiu Chang ${ }^{5}$, Po-Huang Liang ${ }^{10,11}$, Jinn-Moon Yang ${ }^{1,3,12,13^{*}}$

${ }^{1}$ TIGP Bioinformatics Program, Institute of Information Science, Academia Sinica, Taipei 115, Taiwan

${ }^{2}$ Institute of Bioinformatics and Structural Biology, National Tsing Hua University, Hsinchu 300, Taiwan

${ }^{3}$ Institute of Bioinformatics and Systems Biology, National Chiao Tung University, Hsinchu 300, Taiwan

${ }^{4}$ Department of Veterinary Medicine, National Chung Hsing University, Taichung 402, Taiwan

${ }^{5}$ Institute of Preventive Medicine, National Defense Medical Center, New Taipei City 114, Taiwan

${ }^{6}$ National Yang-Ming University, Taipei 112, Taiwan

${ }^{7}$ Graduate Institute of Medical Sciences, National Defense Medical Center, New Taipei City 114, Taiwan

${ }^{8}$ Department of Laboratory Medicine, National Taiwan University Hospital, Taipei 100, Taiwan

${ }^{9}$ National Taiwan University College of Medicine, Taipei 100, Taiwan

${ }^{10}$ Institute of Biological Chemistry, Academia Sinica, Taipei 115, Taiwan

${ }^{11}$ Institute of Biochemical Sciences, National Taiwan University, Taipei 106, Taiwan

${ }^{12}$ Department of Biological Science and Technology, National Chiao Tung University, Hsinchu 300, Taiwan

${ }^{13}$ Center for Intelligent Drug Systems and Smart Bio-devices, National Chiao Tung University, Hsinchu 300, Taiwan

*Corresponding author, Email: moon@ faculty.nctu.edu.tw 
A
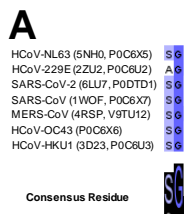

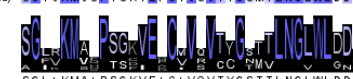
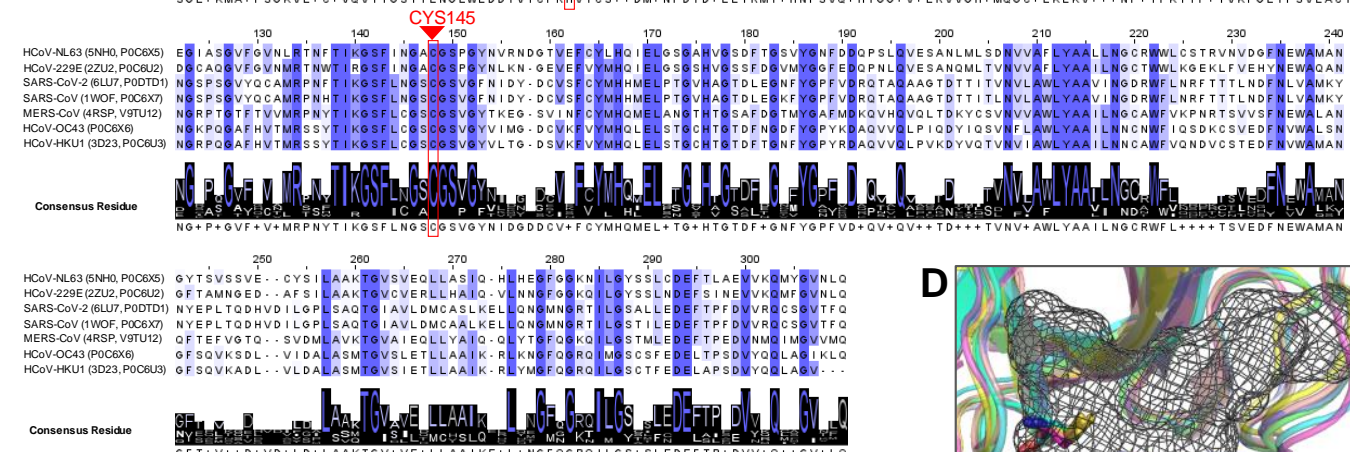

B
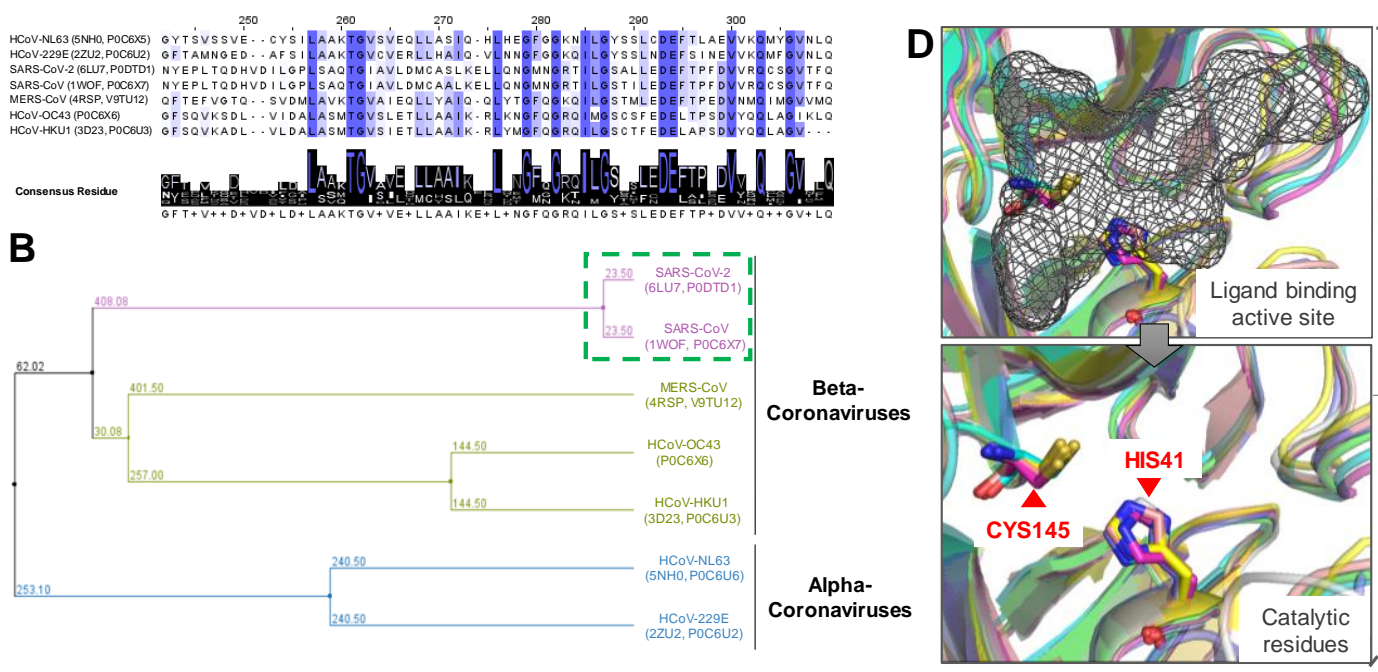

C
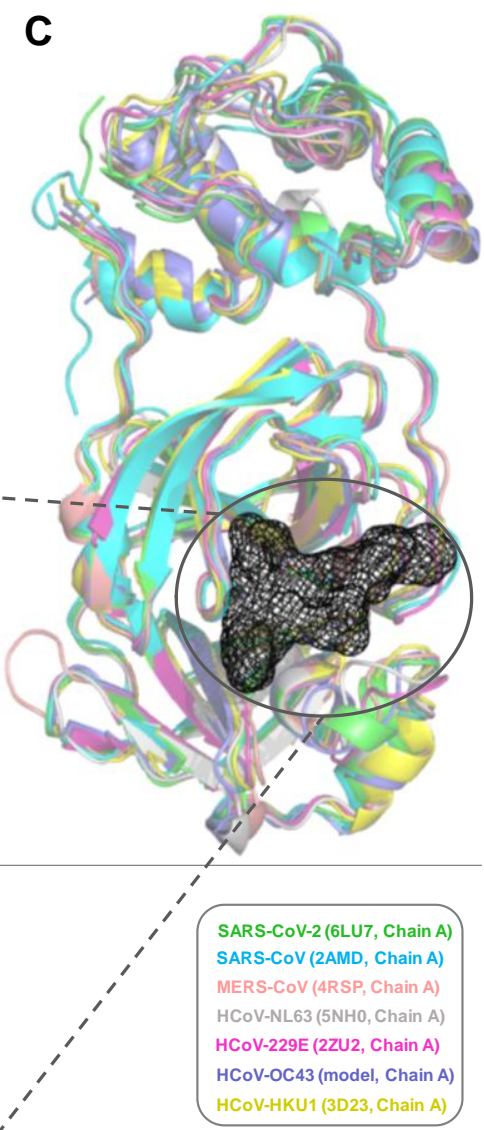

Figure S1. 3CL proteases of human coronaviruses: sequence and structure analysis. (A) Multiple Sequence Alignment (MSA) of 3CL-proteases of human coronaviruses: SARS-CoV-2 (PDB ID: 6LU7, P0DTD1), SARS-CoV (PDB ID: 2A5I, P0C6X7), MERS-CoV (PDB ID: 4RSP, V9TU12), HCoV-HKU1 (PDB ID: 3D23, P0C6U3), HCoV-OC43 (P0C6X6), HCoV-NL63 (PDB ID: 3TLO, P0C6U6), HCoV-229E (PDB ID: 2ZU2, P0C6U2), residues are colored purple by \% conservation, consensus residues are shown, the protease catalytic residues His-41 and Cys-145 are highlighted in red. (B) Phylogenetic trees of the viral proteases showing the alpha/beta coronavirus lineages, closest homologs from SARS-CoV2 and SARS-CoV highlighted by green dotted outline. (C) Structure alignment of the seven 3CL-protease crystal structures (and modeled HCoV-OC43 protease), with protease active sites shown by mesh. (D) The insights show well-aligned ligandbinding active site cavities (mesh) and conserved conformations of catalytic His-41 and Cys-145 residues. 
A SARS-COV-2 (6LU7, PODTD1) 1 SGFRKMAFPSGKVEGCMVQVTCGTTTLNGLWLDDVVYYCPRHVICTSEDML SARS-CoV (2A5I, P0C6X7) 1 SGGRKMAFPSGKVEGCMVQVTCGTTTLNGLWLDDTVYCPRHVICTAEDML

SARS-COV-2 (6LU7, PODTD1) 51 NPNYEDLLIRKSNHMFLVQAGNVQLRVIGHSMQNCVIKGKVDTA TPKTPK

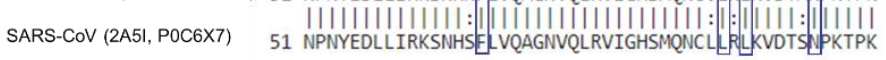

SARS-COV-2 (6LU7, PODTD1)101 YKFVRIQPGQTFSVLACYNGSPSGVYQCAMRPNFTIKGSFLNGSCGSVGF SARS-CoV (2A5I, P0C6X7) 101 YKFVRIQPGQTFSVLACYNGSPSGVYOCAMRPNH IIKGSFLNGSCGSVGF

SARS-COV-2 (6LU7, PODTD1) 151 NIDYDCVSFCYMHHMELPTGVHAGTDLEGNFYGPFVDRQTAQAAGTDTTI SARS-CoV (2A51, POC6X7) | 1 ||||||||||||||||||||||||||||||| |||||||||||||||||||||||

SARS-CoV-2 (6LU7, PODTD1) 201 TVINLAWLYAAVINGDRWFLNRFTTTLNDFNLVAMKYNYEPLTQDHVDIL SARS-CoV (2A51, P0C6X7) 201 TLNVLAWLYAAVINGDRWFLNRFTTTLNDFNLVAMKYNYEPLTQDHVDIL SARS-CoV-2 (6LU7, PODTD1) 251 GPLSAQTGIAVLDMCAS[KELLLNGMNGRTILGSALIEDEFTPFDWRQC SARS-CoV (2A51, POC6X7) 251 || || || || || || || || |||||||||||||||||| . |||||||||||| $\mid$ SARS-COV-2 (6LU7, PODTD1) 301 SGVTFP

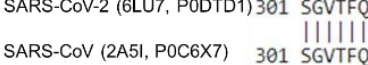

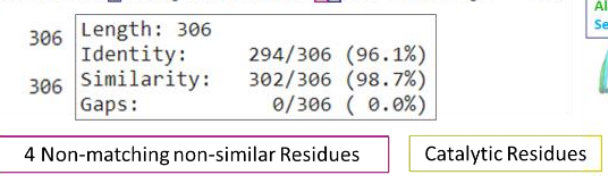

4 Non-matching non-similar Residues

Catalytic Residues

\footnotetext{
8 Non-matching similar Residues
}

B
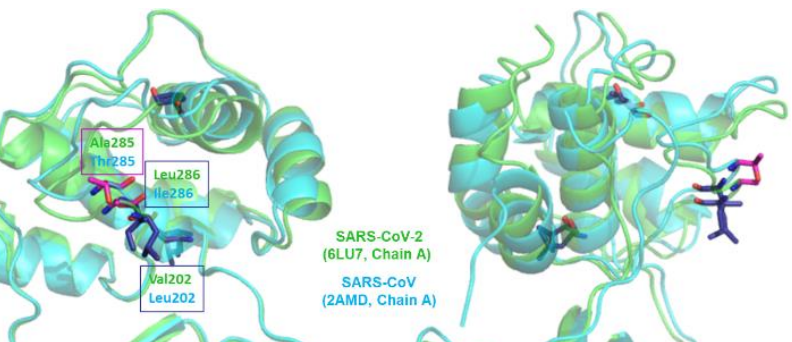

Figure S2. Pairwise sequence and structure comparison of SARS-CoV-2 and SARS-CoV 3CL proteases. (A) Pairwise sequence alignment of SARS-CoV-2 (PDB ID: 6LU7, P0DTD1), SARS-CoV (PDB ID: 2AMD, P0C6X7) 3CL proteases with $\%$ identity and similarity, showing outlined non-matching (similar: blue, non-similar: magenta) and catalytic residues (yellow). (B) Structural alignment of these proteases showing the non-matching similar and non-similar residues (as sticks) along with the active site cavity (grey surface), the $90^{\circ}$ view highlighting the non-matching similar residue Ser-46-Ala-46 (bold blue outline). 


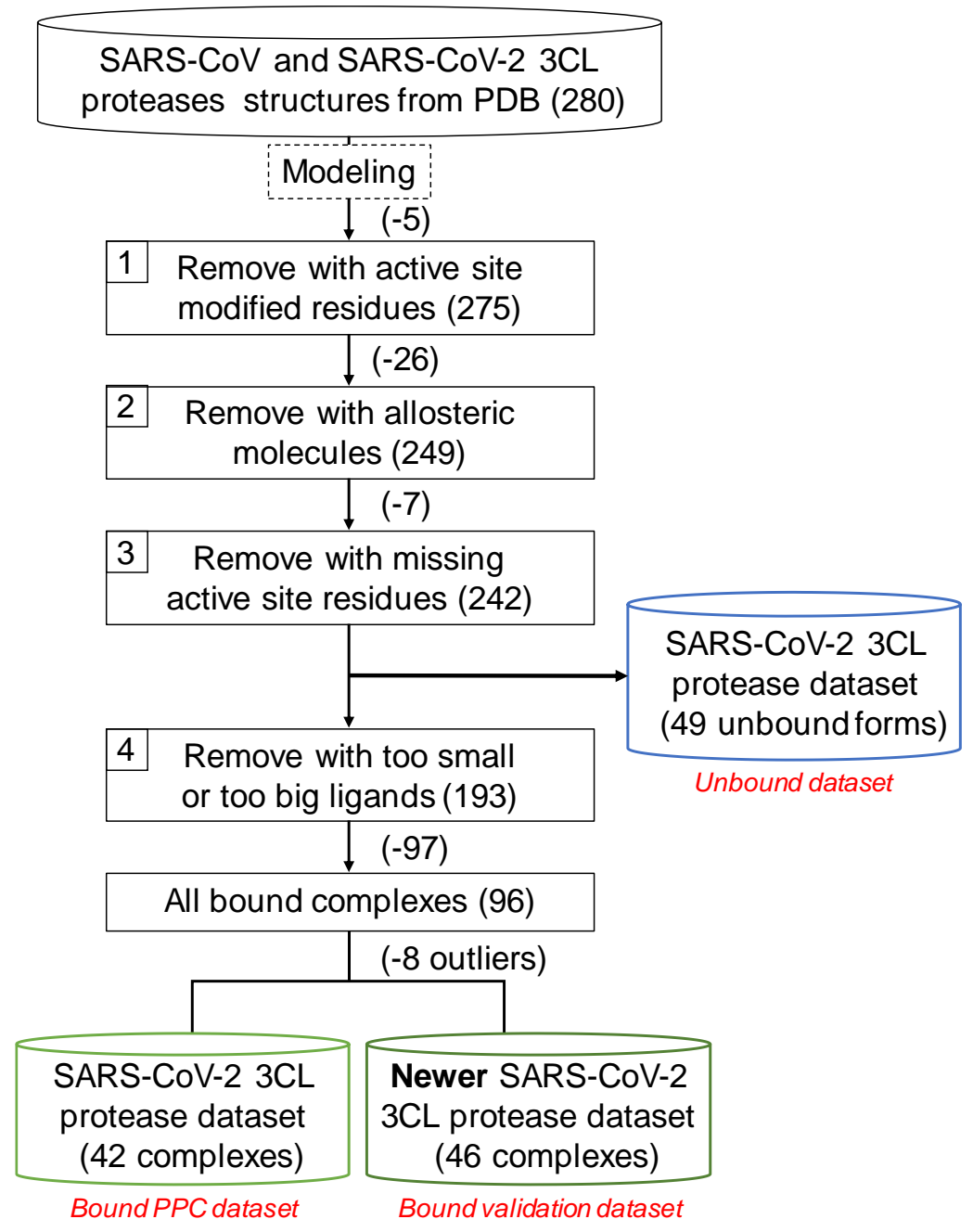

Figure S3: Summary for collection of SARS-CoV-2 3CL protease datasets. We collected a total of 280 SARSCoV/CoV-2 3CL protease crystal structures available, and created their SARS-CoV-2 models. Stepwise analysis was performed at each step, removing the unsuitable structures: 1) active sites with modified residues, 2) allosteric molecule induced perturbated active sites, 3) active sites with missing residues, 4) too large/small ligands with too many/few interactions. The extracted active sites of the filtered structures with or without ligands were assigned into 3 major datasets: 42 bound structures for identifying PPCs, 46 newer bound structures for validation and 49 unbound structures for comparative analysis. 


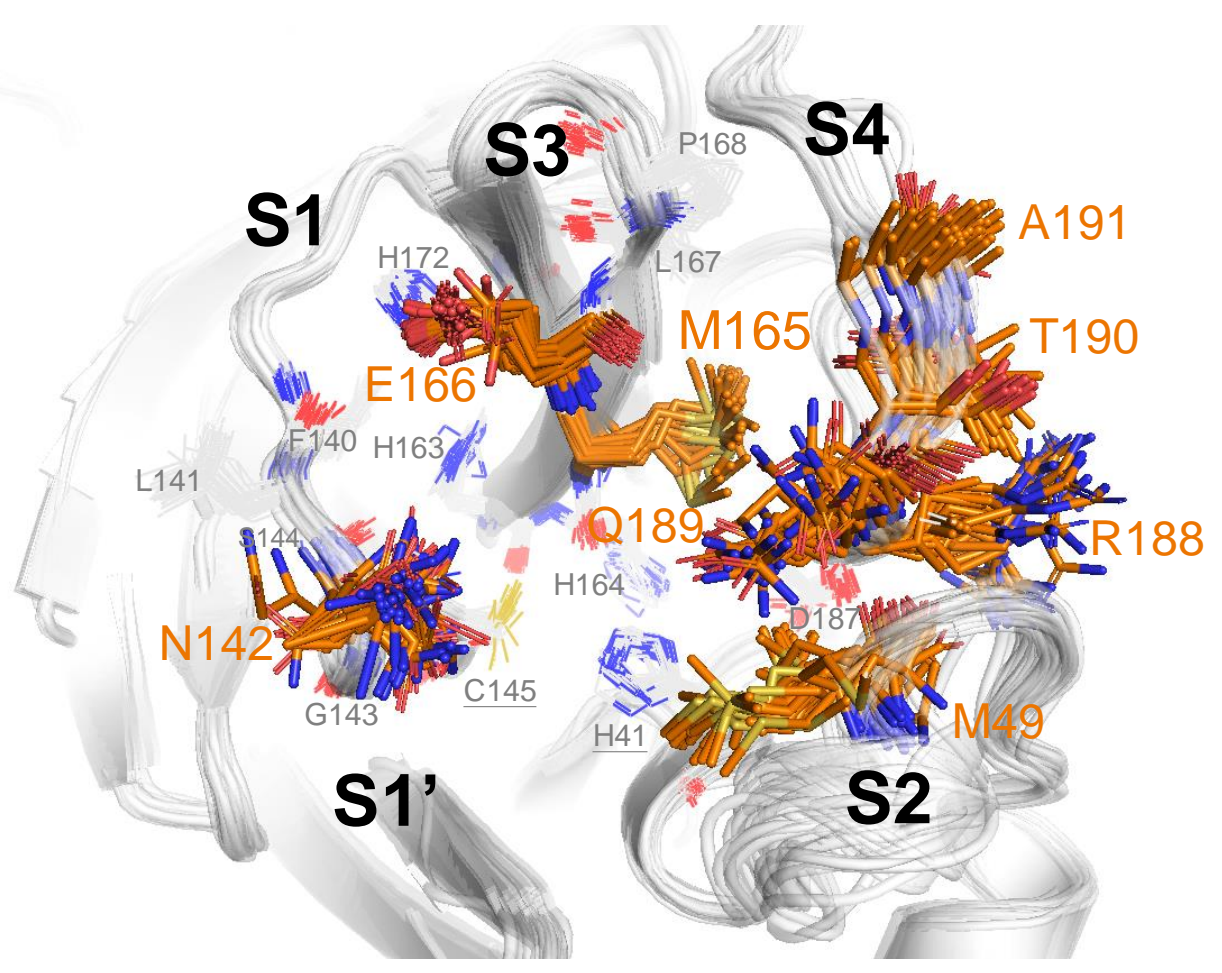

Figure S4. SARS-CoV-2 3CL proteases: active sites and residue conformations. The 42 3CL protease structures from the protease dataset were aligned and are shown (grey cartoon and lines) with labeled active site residues (grey label) along with catalytic residues (underlined label). At each of the active site subpockets S1' to S4, the residues with the variable main chain and side chain conformations which were specifically highlighted with orange sticks and labels.

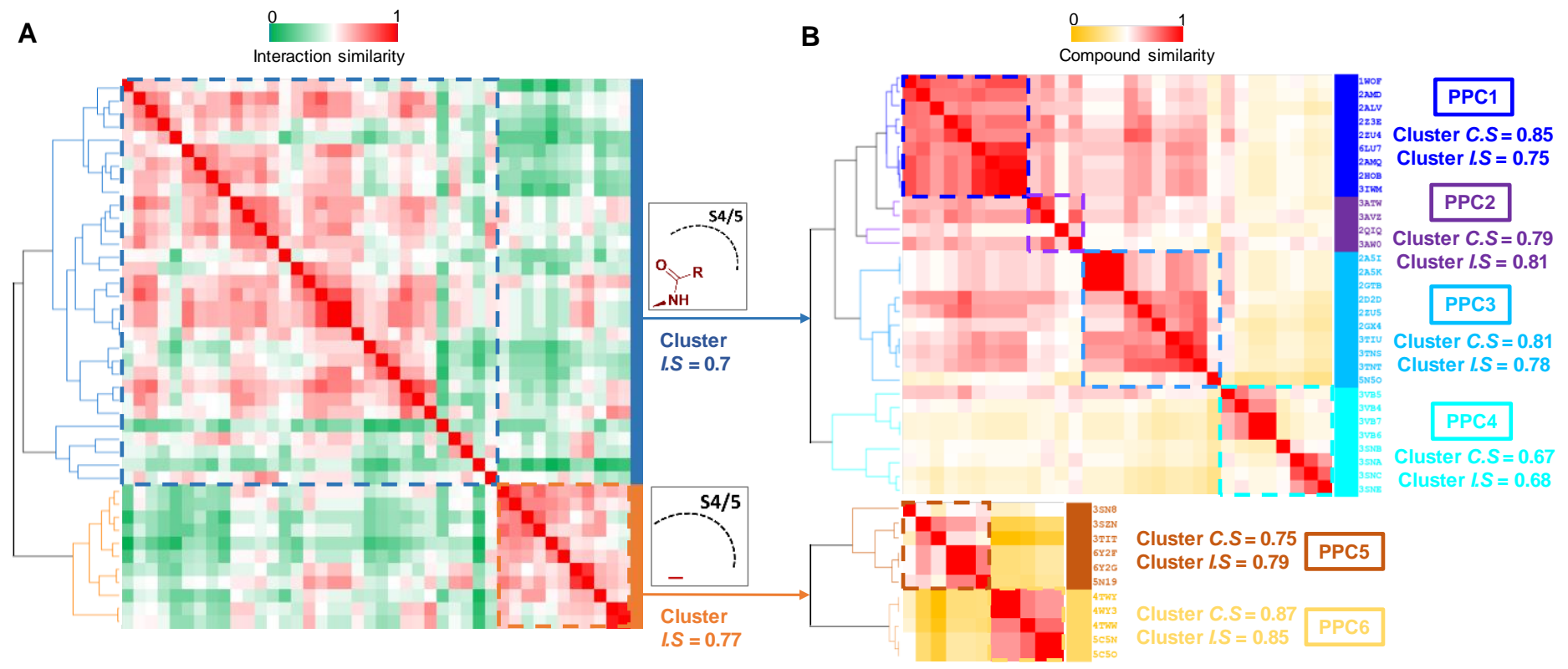

Figure S5. Two-Stage Combinatorial Clustering (TSCC). (A) In the first stage, the 42 protease-ligand complexes were subjected to Two-way hierarchical clustering based on Interaction Similarity (I.S) scores (0 to 1) shown as a heatmap. The identified blue cluster with 32 complexes and the orange cluster with 11 complexes are outlined and the cluster I.S values shown (cluster I.S > 0.66 - significant interaction similarity within the cluster), the major difference between clusters in S4 subpocket occupancies is depicted. (B) In the second stage, each of blue and orange clusters was clustered by ligand Compound Similarity (C.S) scores (0 to 1) shown in heat maps, resulting in 6 distinct clusters called PPCs (mean cluster C.S/I.S > 0.66 - significant compound/interaction structure similarity within the cluster) with cluster I.S and C.S values. 


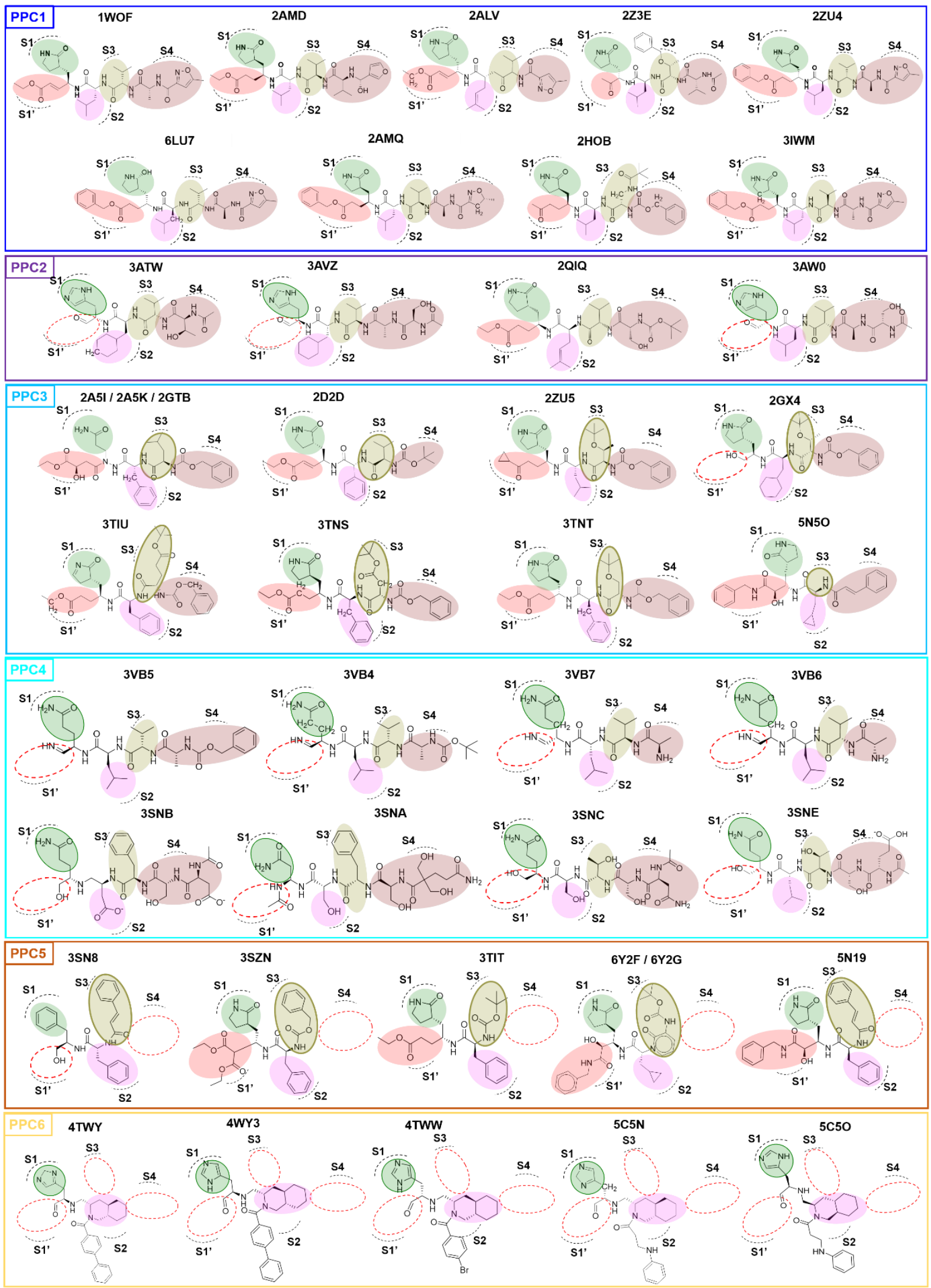

Figure S6. 6 Protease Pharmacophore clusters (PPCs) and 42 protein-ligand complexes. For all PPC members of the protease dataset, 2D structures show ligand moieties (highlighted by the moiety colors used in Figure 2) occupying PPC active site subpockets. 


\section{Validation of PPCs by unbound protease datasets:}

For verifying our discovered PPCs, we used unbound form 3CL proteases and also studied their similarities and differences with our ligand-bound PPCs. An unbound dataset containing 49 structures was systematically collected from $\sim 280$ protease structures as shown in Figure S3. We then comparatively analyzed these unbound protease structures, to check if the unbound protease active sites were similar to those of the current ligand bound PPCs, and if could be assigned to them. A residue RMSD metric-based approach was used, instead of the TSCC approach, as the unbound forms lacked ligand structures and interactions, essential for calculating the C.S and I.S scores. We first calculated the anchor residue intra/inter-PPC RMSD (maximum allowed) values for each of 6 PPCs (Figure 7A) which separated at $2.5 \AA$ verifying our distinct PPCs and giving an RMSD cutoff of $2.5 \AA$ to assigning structures into PPCs. Then, for each new unbound protease structure we calculated the residue RMSD with 42 PPC members and assign it to specific PPC, with which it has the lowest RMSD value $<2.5 \AA$ cutoff, ensuring its average RMSD distance with all PPC members within the cutoff. Thus we successfully assigned $\sim 80 \%$ (39 structures) of the unbound structures (Figure S7B), 4 structures to PPC1, 2 structures to PPC3, 7 structures to PPC4, 24 structures to PPC5 and 2 structures to PPC6 respectively, while 10 structures could not be assigned to any PPC. This points out that many of the current unbound structures show a high degree of overall active site similarity and the same conformations states as some of our PPCs. The active sites of the unbound forms 3E91 of PPC4 and 3EA8 of PPC5 when aligned to representatives of PPC4 (3VB6) and PPC5 (3TIT) respectively (as in Figure S7C), clearly show matching conformations evident by their intra-PPC4 RMSD of $1.63 \AA$ and an intra-PPC5 RMSD of $0.56 \AA$.

We then explored flexibilities of active sites residues in unbound forms by analyzing anchor residue (main chain and side chain) RMSDs in 39 unbound PPC-assigned structures compared to the bound PPCs, the results showed a high Pearson Correlation Coefficient (PCC) of 0.95 (main-chain) and 0.83 (side-chain) (Figure S7D). The strong correlation of main chains indicates stable overall structure with no major protease conformational change in ligand bound forms, while moderate correlation of side chains indicate some possible differences in residue side chain flexibilities amongst both forms (like for the M49 residue). Similar conclusions of no major global perturbations upon ligand binding, but local subtle differences in amino acid side chain flexibilities between both forms, have been deduced in studies using MD simulation of $3 \mathrm{CL}$ protease bound and unbound forms ${ }^{1}$. This strengthens our observations of unbound forms having PPC states. We further investigated the side chain flexibilities, for each of the anchor residues in unbound (39) vs bound PPCs (42) (Figure S7E). Firstly we observed, common rigid residues in both unbound and bound PPCs like H41, C145 (both catalytic), H163, H172, F140, G143, H164, S144, D187, etc. The residues M165, L167, P168 along with L141 are more flexible in unbound structures than bound form (> $1 \AA$ A purple arrow), conversely M49 seen as the most flexible residue showed higher flexibility in bound forms. 

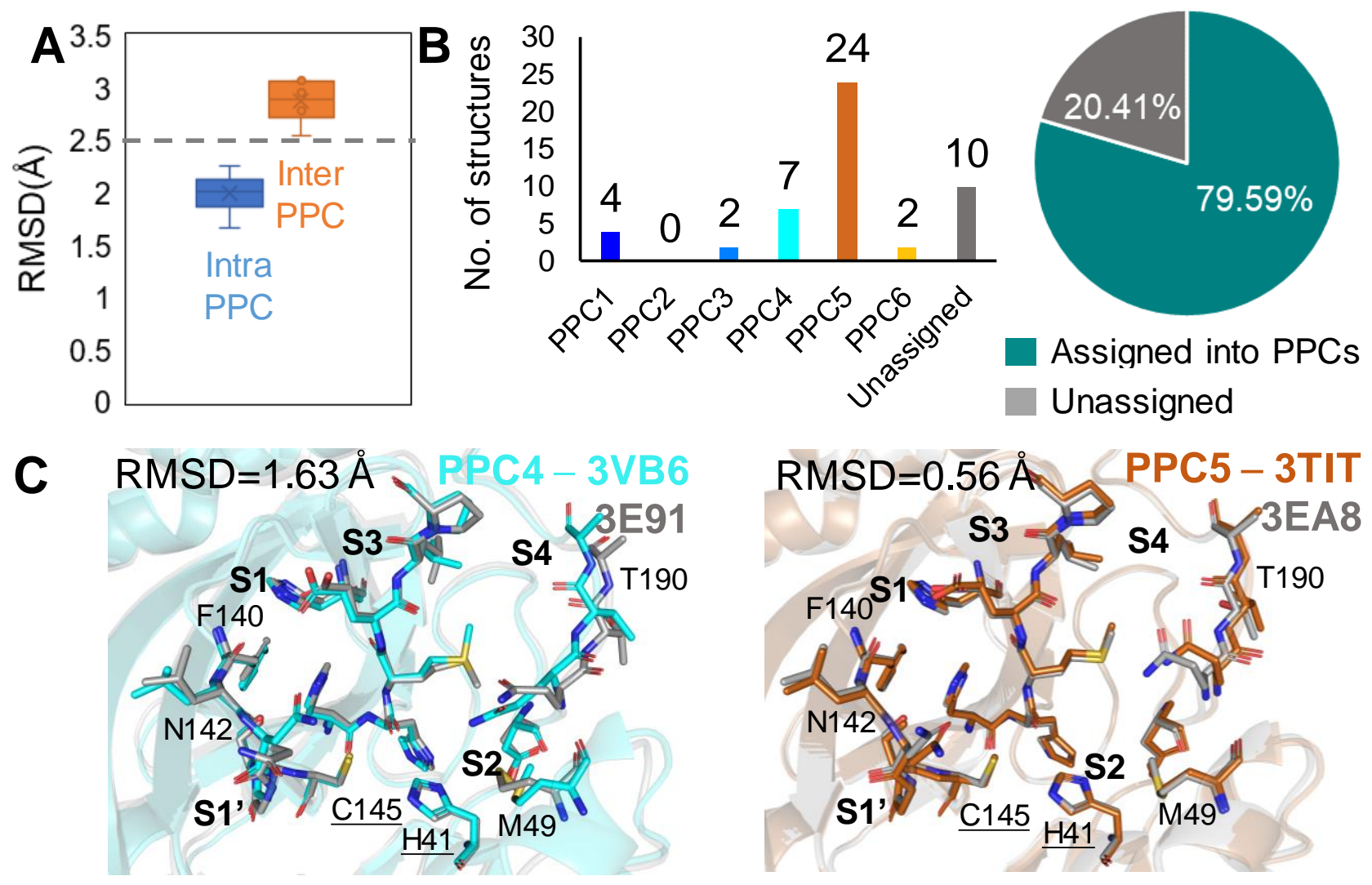

D

E

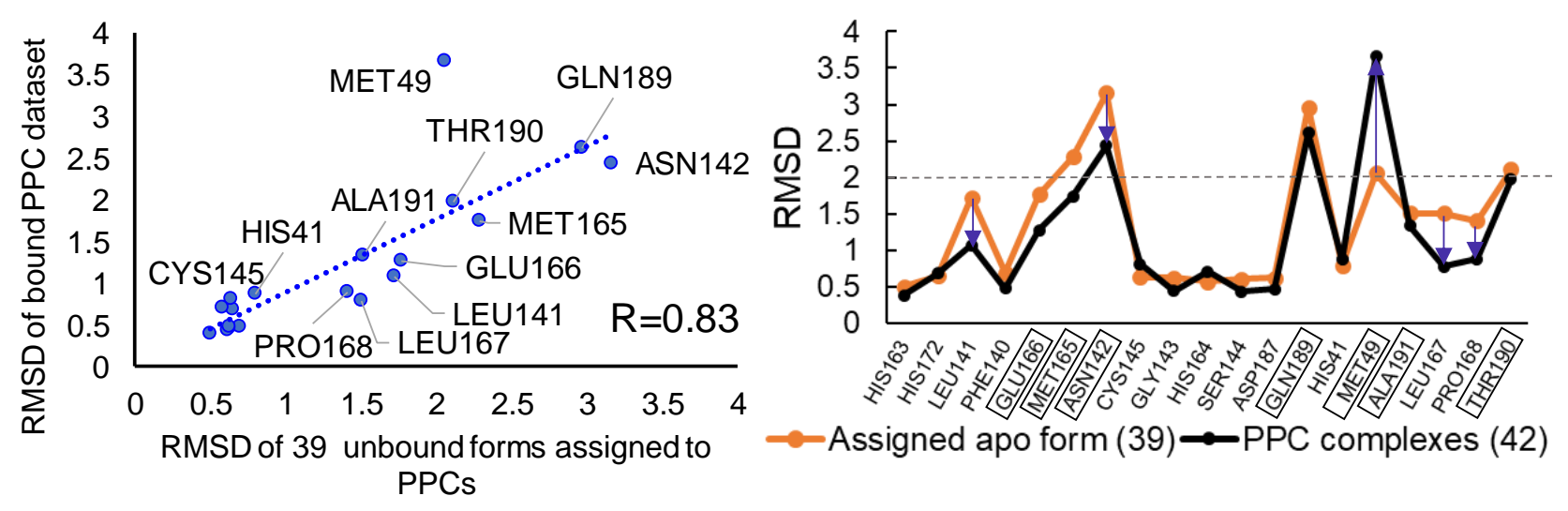

Figure S7. Validation of PPCs by unbound proteases. (A) Calculation of intra/inter-PPC RMSDs of anchor residues for our 6 PPCs and their distribution identifies $2.5 \AA$, as the RMSD residue cutoff amongst PPCs. (B) Statistical analysis of 49 SARS and SARS-COV-2 protease unbound structures. 39/49 (80\%) structures were assigned by RMSD metric to PPC5(24), PPC4(7), PPC1(4), PPC3(2) and PPC6(2 structures), if the RMSD between new structures and the original structures in PCCs. (C) Structure comparison of assigned unbound structures, PPC4 representative structure 3VB6(cyan) and apo-form structure 3E91(gray), RMSD of 19 anchor residue side chain is $1.63 \AA$ A. PPC5 representative structure 3TIT(brown) and unbound structure 3EA8(gray). RMSD of 19 anchor residue side chain is $0.56 \AA$. (D) Pearson correlation coefficient calculated using anchor residue sidechain RMSDs, between 39 unbound PPC and 42 bound PPC structures were plotted, showing an overall PCC correlation of 0.83, with many of the residues showing RMSD correlations in both forms. (E) Analysis of each of these anchor residue RMSDs in both forms were compared. Purple arrows highlights the residues that show > 1 A RMSD difference, outlined flexible residues. 
On the other hand, the 10 unassigned unbound structures (20\%) were further clustered based on the RMSD metric as shown in Figure S8A showing one new cluster (green). All these 10 unbound unassigned structures have unrealistic active sites with a large RMSD (> $2.5 \AA$ ), due to three factors (Figure S8A): (1) an active site with pH-induced conformational change (PDBs: 2BX3, 2BX4, and 2GT8); (2) a mutation in the active site (PDBs: 3F9F and 3F9H); and (3) an inactive monomer structure (PDBs: 2PWX, 2QCY, 3F9E, and 3M3T). Among these, for the example case 3F9E, the active site structure was compared to PPC1 representative 6LU7 (Figure S8B). A closer observation reveals major movements of subpocket loop residues, F140, N142, M49 and M165 (RMSD 4-9 ̊), distorting the S1 subpocket binding environment unlike any of the PPCs (Figure S8C).

A

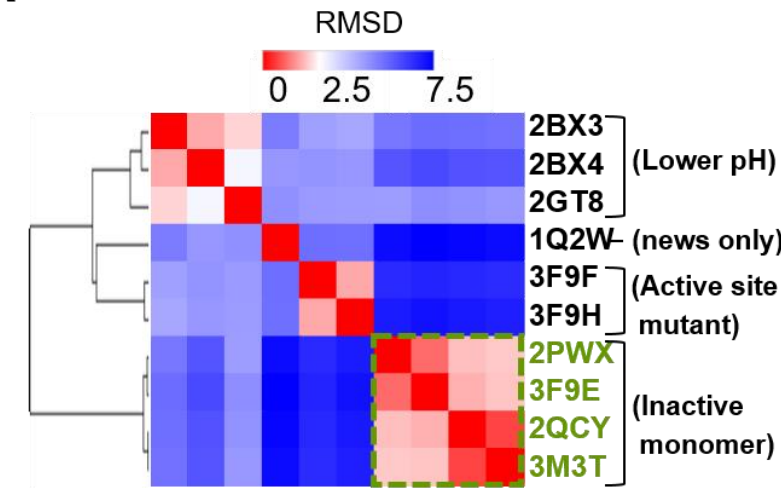

C

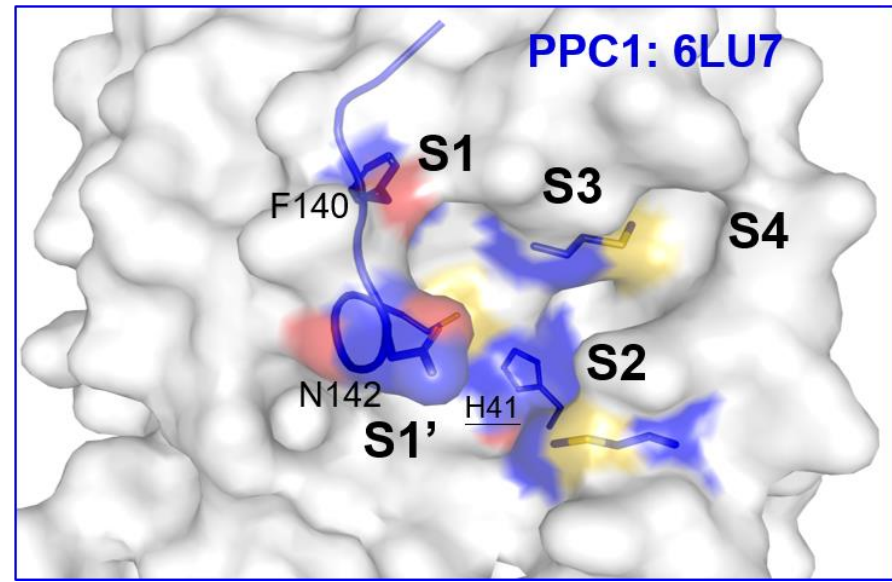

B
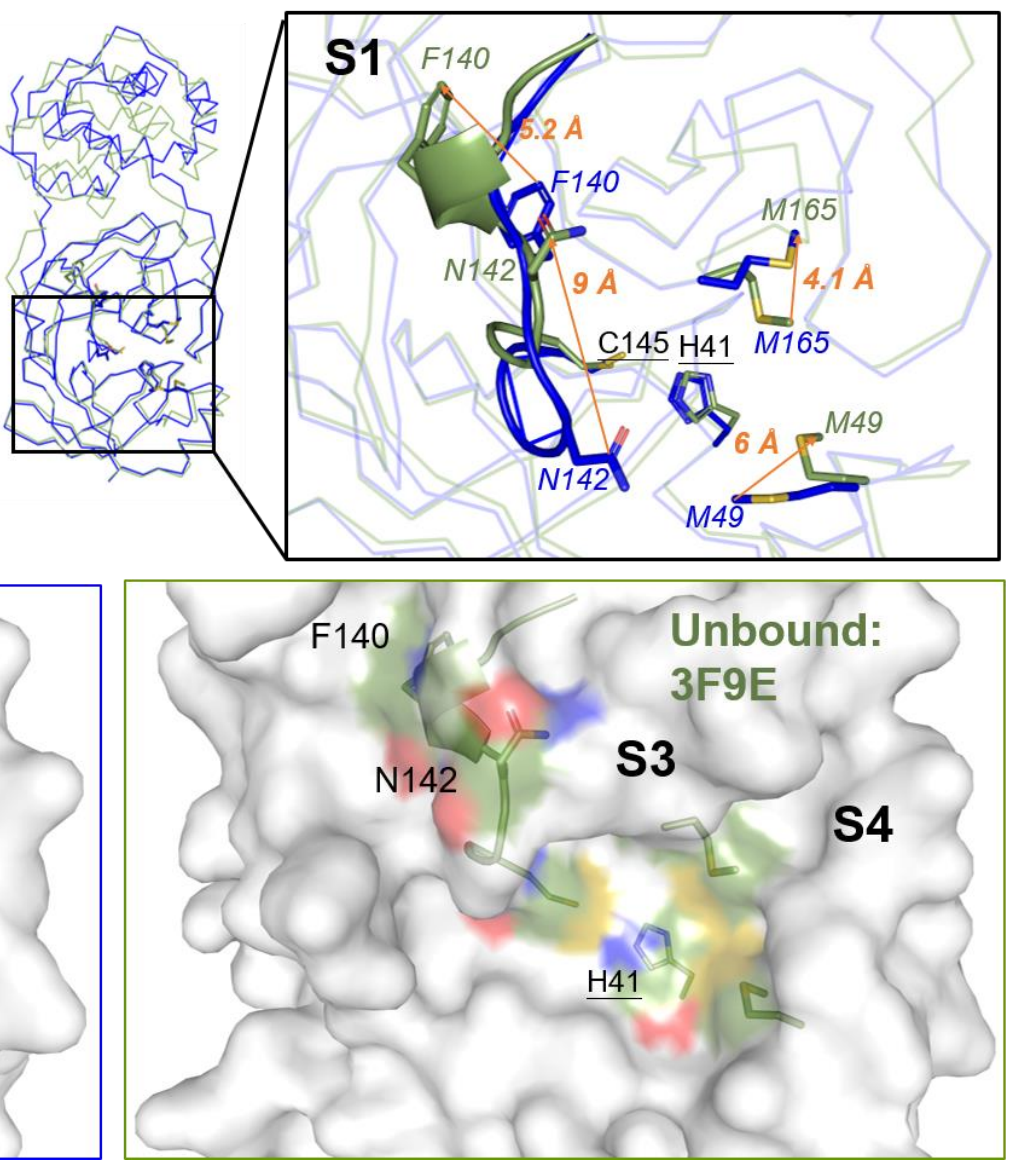

Figure S8: Unassigned unbound proteases. (A) RMSD-metric based clustering for unassigned unbound forms showing 10 unassigned structures. (B) New cluster representative 3F9E structure (green) aligned to bound PPC1: 6LU7 (blue) with insight showing differential S1 subpocket with loop conformational change and movement of residues F140, N142, M49 and M165 (C) unassigned 3F9E vs PPC1: 6LU7 protein surfaces showing the distorted S1 subpocket in the unbound form. 


\section{Validation of PPCs by bound protease datasets:}

The rapid moving pace of SARS-COV-2 research led to the discovery of newer ligand bound protease structures, using which a ligand bound protease dataset with 46 structures was prepared for verifying the PPCs obtained from the TSCC method (Figure S9). For this, we calculated the similarity scores C.S and I.S from the TSCC method for each of the new ligand bound protease structures, and their average C.S and I.S scores with each of the 6 PPCs. Then by considering the cluster I.S and C.S scores of our PPCs (all values > 0.6), we assigned each new bound structure to the PPC with which it had the highest average C.S and I.S score, both of which must be $>0.6$ cutoffs. As a result, the $89 \%$ of the 46 new bound structures were assigned to 6 PPCs: PPC1 (4), PPC2 (2), PPC3 (14), PPC4 (3), PPC5 (17) and PPC6 (1 structure) and 5 bound forms (11\%) remaining unassigned (as summarized in Figure S9A). We further comparatively analyzed the assigned new bound protease structures alongside our PPCs, to verify their relevance to the current PPCs. Figure S9B shows the aligned new bound form PPC structures assigned to PPCs with the original PPC representatives. We observed the perfect alignment of the new bound form ligands with the PPC representative ligands and also the overall active site residue conformations. For example, 6XBG ligand structure contains colored moieties similar to the PPC3 ligand (at the S1, S2, S3 and S4 subpocket binding groups) with well aligned binding poses and similar conformation of the interacting residues. As a result, the overall active site cavity (shown as surface) are also similar, justifying the new PPC assignment. Similar patterns are observed for other PPC5, 6XBH is similar to the member of PPC5 (3SZN) and with high C.S and I.S. In contrast, we also examined the unclassified newer structure active sites, that couldn't be assigned to PPCs (Figure S9C). For instance, in the 6XFN structure although the ligand shows good compound similarity (avg, cluster C.S $>0.6$ cutoff) with all the 6 PPC ligands, fails to bind properly extend its P1-P4 groups into the respective S1-S4 subpockets, showing significant interaction differences (I.S $<0.6$ cutoff).

This kind of structures representing the crystal structural anomalies have been successfully discarded from the PPCs by the C.S/I.S scores used in our TSCC method. For another kind of newer structures that are PPC outliers like 6XFN (low I.S) and 7JU7 (low C.S and I.S) the ligand structure and its interaction are both significantly dissimilar with that of the PPCs (Figure S9C). The rightful assignment of $89 \%$ of newer structures to PPCs reconfirms the coverage of our PPCs and the robustness of our method, also eliminating fewer outliers which are either crystal anomalies and rarely seen interaction patterns by unique uncommon ligands. Findings from the study of our verification analysis, strengthen our beliefs that our identified PPCs can encompass the majority of the existing SARS-CoV-2 3CL protease conformational states in many of the $\sim 280$ bound and unbound form crystal structures. Moreover, the adaptability, application and extension of our PPCs with newer crystal structures are demonstrated. In summary, the newer datasets validate the robustness of our PPCs and scalability of our approach to accommodate and explain the newly crystallized structures and that will be crystallized in the future. 


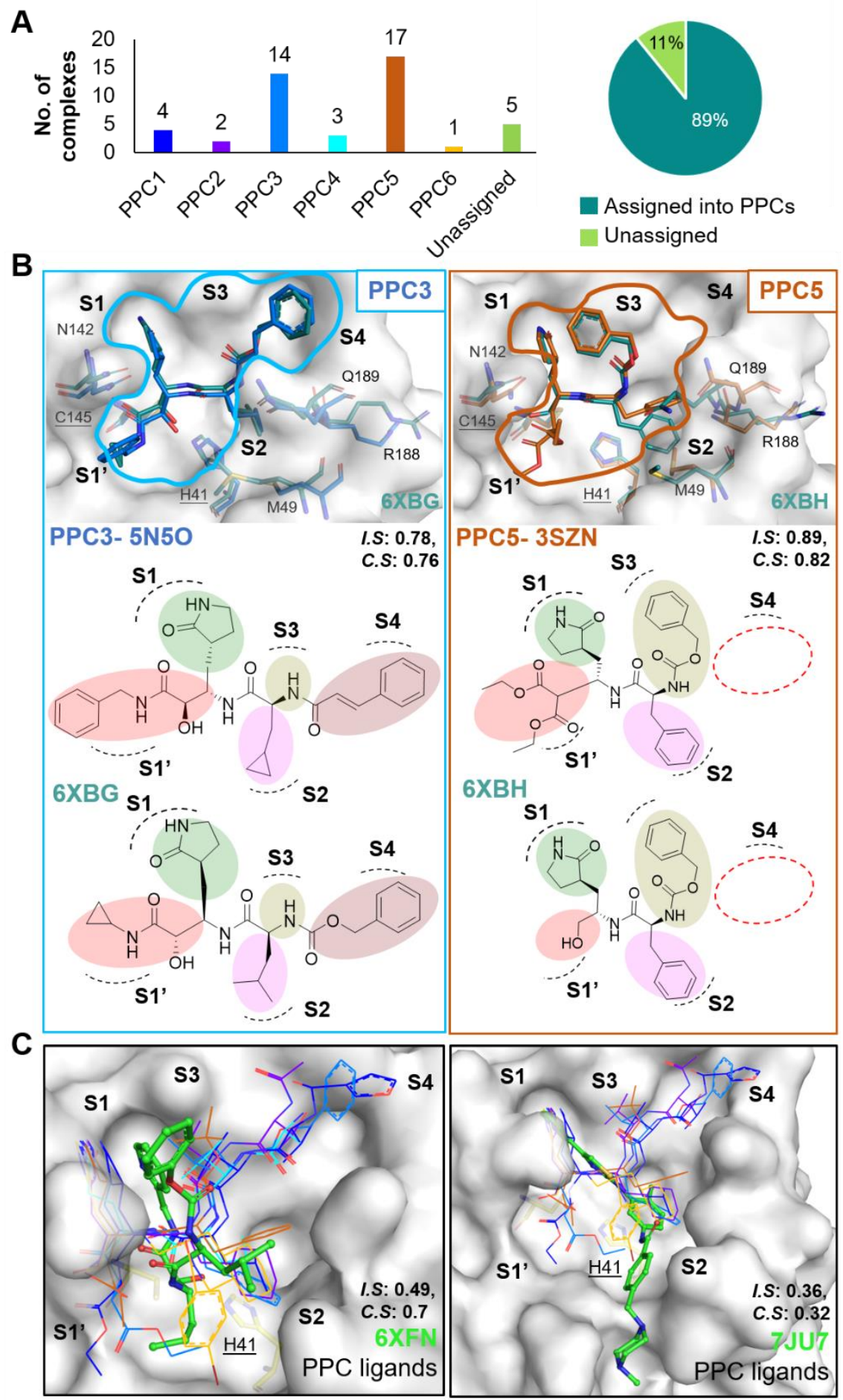

Figure S9. Analysis of PPCs and new bound protease structures. (A) Statistic analysis of newer bound SARS-CoV and SARS-CoV-2 protease complexes assigned into PPCs. The assignment was based on the product of I.S and C.S scores from TSCC, both of which satisfied the cutoff $>0.6$ and the maximum similarity with that PPC. The graph and the pie-chart indicated that $89 \%$ (41/46) new bound structures can be assigned to anyone of six original PPCs with 5 unassigned newer complexes. (B) Structural analysis of PPC-assigned newer bound proteases (colored dark turquoise) showing ligand structures and binding with the PPC structures and ligands. C) Analysis of PPC-unassigned newer bound complexes with PPC ligands unassigned ligands shown as green colored ball and sticks binding to protease shown as white surface. The PPCs representative ligands were shown as lines for reference. The average C.S and I.S score of these unassigned complexed with the all members of that PPCs are shown. 


\section{Verification of PPC anchors by substrate binding mechanisms:}

For pursuing validation of our PPC anchors and their role and involvement in binding mechanisms, we proceeded to use the SARS-CoV-2 Substrate peptides which are known to bind to the protease active site. For this, we explored SARSCoV-2 substrate binding by collecting its substrate peptide residue composition, $\sim 25$ substrates/mimic-bound protease structures (all peptide like compounds collected from the 88 ligand bound protease set) and combining it with our identified PPC anchors (Figure S10). We thus investigated the substrate P1-P4 peptides binding to S1-S4 subpockets respectively and the results show our PPC anchors through anchor residues and H-V interactions, and anchor moiety preferences, can elucidate peptide residue binding and some preferences. First, a total of 11 SARS-CoV-2 substrate sequences occurring in between different protein sequences in its polyprotein (see Figure S10A) were collected, only highlighted residues have crystal structures and were explored. The variance of residues at each of these positions was analyzed using WebLogo to see all substrates only preferring Gln (Q) at the P1 position, while multiple residues at other positions. The results in Figure S10B, highlights each substrate peptide residues binding at its respective subpockets occupying PPC anchors (in reference PPC1 cavity). For the conserved substrate P1 'Gln' occupies core EHV2 with its $-\mathrm{CO}-\mathrm{NH}_{2}$ group (top $3^{\text {rd }}$ prefer anchor $\mathrm{H}-$ moiety) is fixed in the deep S1 subpocket via H-bonding with F140 and H163 anchor residues and van der Waals interactions with F140, E166 anchor residues via its alkyl chain (top $3^{\text {rd }}$ preferred anchor V-moiety), repeating across 8 complex structures. The Gln main chain mainly occupies the HV1 anchor with the backbone -NH- forming hydrogen bonding interactions with the anchor residue H164. Similarly, at S2 subpocket, the P2 'Leu/Phe' residues consistently engage the core anchor V3 with preferences for hydrophobic moieties (in Figure S10B). These S1, S2 subpocket core anchors define the conserved interaction environments favoring defined moieties, for conserved P1 and similar P2 residues. The anchors at S3 and S4 subpockets offer interactions but accommodate some variability in P3 and P4 substrate residues, respectively. Exploring S3 subpocket, we find anchors HV5 and H6 guiding the binding of Val and Thr residues, where the main chains are stabilized by H-bonding interactions with $\mathrm{H} 6$ anchor resides like E166. The P3 side chain occupies the mixed HV5 anchors supports both H-bonding interactions of Thr side chains and also alkyl side chains of Val (in Figure S10B). The P4 residue is stabilized at the $\mathrm{S} 4$ subpocket via $\mathrm{H} 6$ that offer $\mathrm{H}$-bonding interactions with the $\mathrm{A}, \mathrm{V}$, T main chains and van der Waals interactions via V6 anchor fixing with all hydrophobic side chains. In summary, our PPC core and consensus anchors can explain binding of several conserved and variable P1-P4 substrate residues and can be considered to be significant. 
\begin{tabular}{cccccc|c}
$\begin{array}{c}\text { Substrate } \\
\text { sequences }\end{array}$ & Substrate cleavage motifs & P4 & P3 & P2 & P1 & P1'
\end{tabular}

RQCSGVTFQSAVKRTIKG (nsp5/nsp6, C-terminus of 3CLpro) TSITSAVLQSGFRKMAFP (nsp4/nsp5, N-terminus of 3CLpro) PCIKVATVQSKMSDVKCT MLDNRATLQAIASEFSSL RANSAVKLQNNELSPVAL SLAATVRLQAGNATEVPA DQLREPMLQSADAQSFLN MYTPHTVLQAVGACVLCN PRRNVATLQAENVTGLFK LWNTFTRLOSLENVAFNV VETFYPKLQSSQAWQPGV

(nsp6/nsp7)
(nsp7/nsp8)
(nsp8/nsp9)
(nsp9/nsp10)

(nsp10/nsp11, nsp10/nsp12) (nsp7/nsp8) (nsp7/nsp8) (nsp7/nsp8) (nsp7/nsp8)
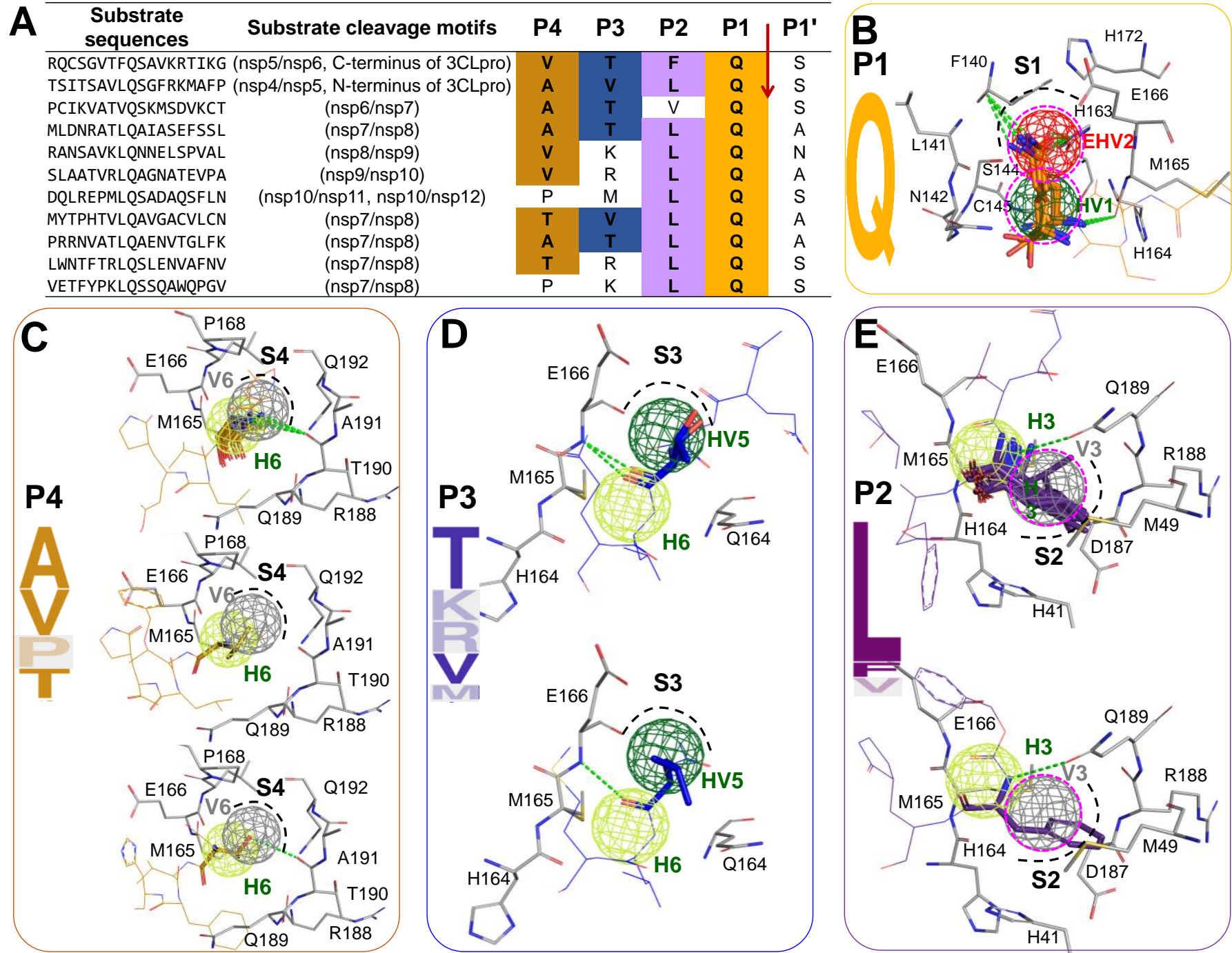

Figure S10: PPC anchors in SARS-CoV 3CL protease substrate binding. (A) The 11 substrate sequences cleaved by the SARS-CoV-2 3CLpro in the polyprotein. Residues crystallized in bound proteases highlighted, residues whose binding mechanisms are analyzed are outlined. (B)-(E) Binding models of substrate P1, P2, P3, and P4 residues at each of the protease S1, S2, S3, and S4 subpockets respectively, involved functional group interactions with anchor residues thus engaging the PPC anchors. The consensus P1'-P1-P4 substrate peptide residues, analyzed by using WebLogo version 2.8.2 (weblogo.berkeley.edu/). 


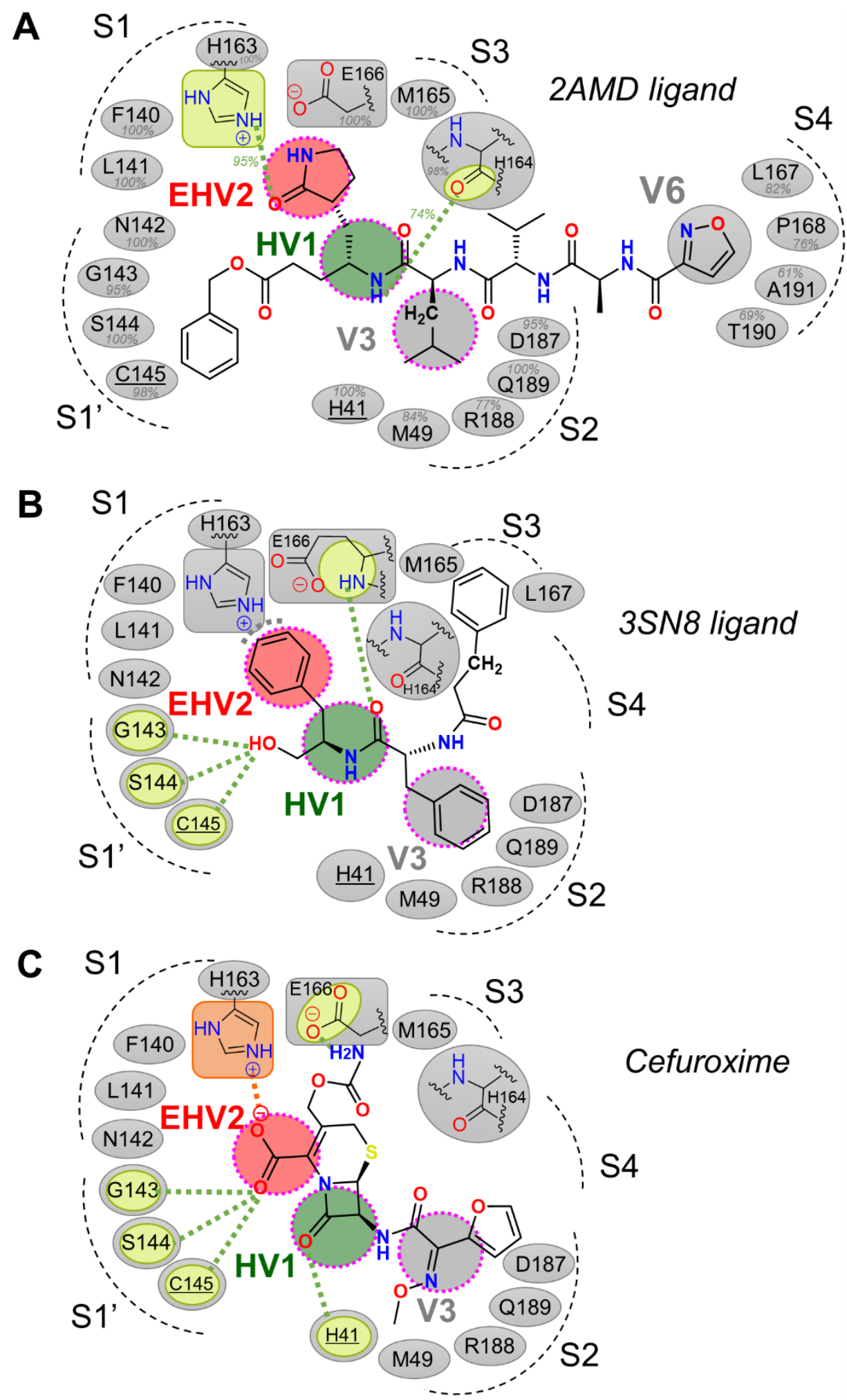

Figure S11. PPC core anchor interaction analysis. The core anchors EHV2, HV1, V3 and their E, H \& V interactions with core anchor residues (residues with their charges are shown by shapes, interactions as dotted lines, all colored by $\mathrm{E}-$ orange, $\mathrm{H}$ - light green, $\mathrm{V}$ - grey) (A) For 42 ligand complexes, the \% interaction frequencies of core anchor interactions via anchors are shown using an example 2AMD ligand (a non-core anchor V6 also included for comparison). Consider the example of complexed 2AMD ligand, where an H-bond acceptor group at the S1 subpocket leads to core anchor residue H163 mediated H-bonding (also found in 95\% of bound ligands). (B) Consider another example of complexed 3SN8 ligand 
with an aromatic benzene ring at S1 leading to strong van der Waals interactions with H163 of the EHV2 core anchor, other interactions at respective subpockets are also displayed. (C) For further studying the core anchor interactions, we analyzed binding of the screened drug Cefuroxime with a negatively charged $-\mathrm{COOH}$ moiety at $\mathrm{S} 1$ which was observed to form electrostatic interactions with the positive charged His 163 side chain, satisfying the core EHV2 anchor.
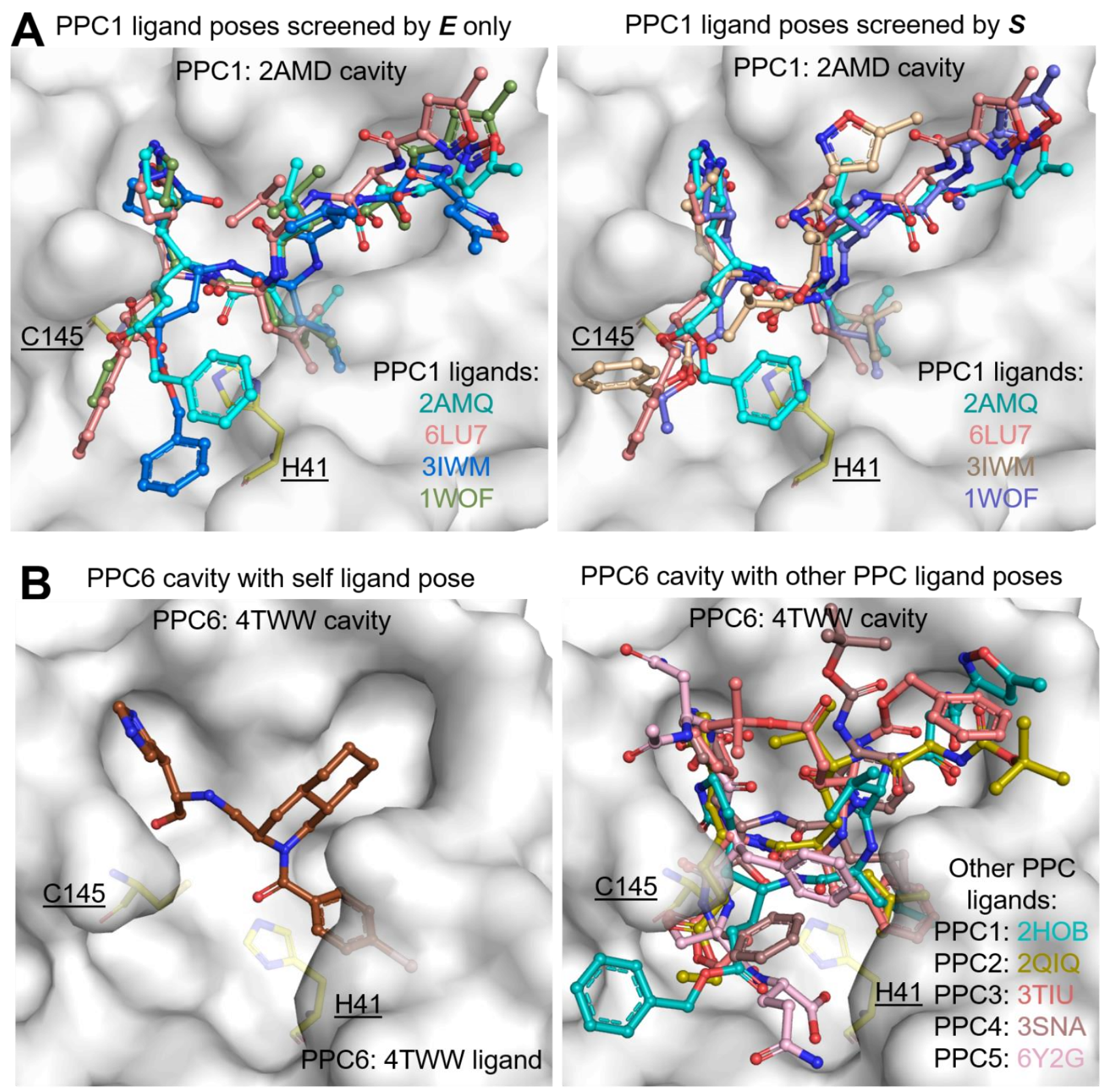

Figure S12. PPCs during performance analysis - PPC1: 2AMD and PPC6: 4TWW. (A) For, PPC1 similar screening performance of PPC Scoring S and energy only E is explored using screened ligand poses, many top-scoring poses also have the best energy and share similar binding poses. (B) For PPC6, while the same ligand occupies some subpockets in the 4TWW cavity, other ligands extend into every subpocket showing better energy, higher score $\mathrm{S}$ and a better hit rate than that of the self-ligand. 
Lopinavir in PPC1

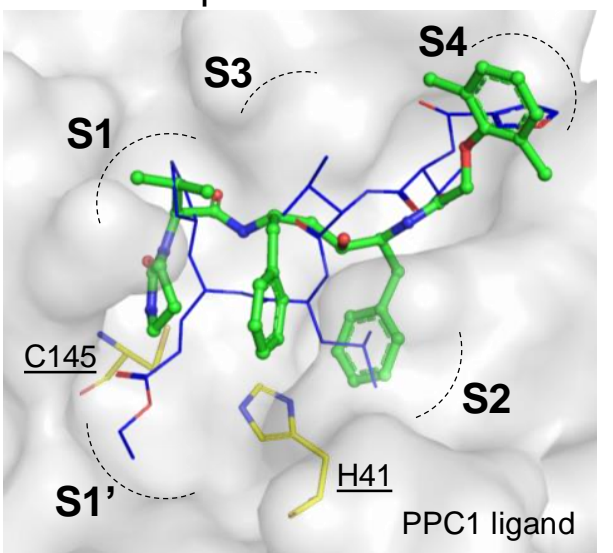

Boceprevir in PPC1

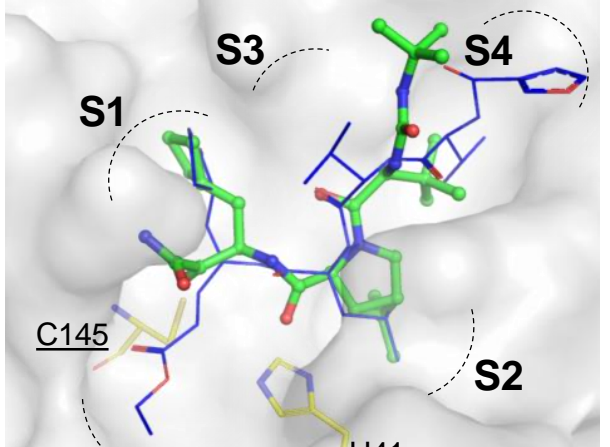

s1
Saquinavir in PPC4

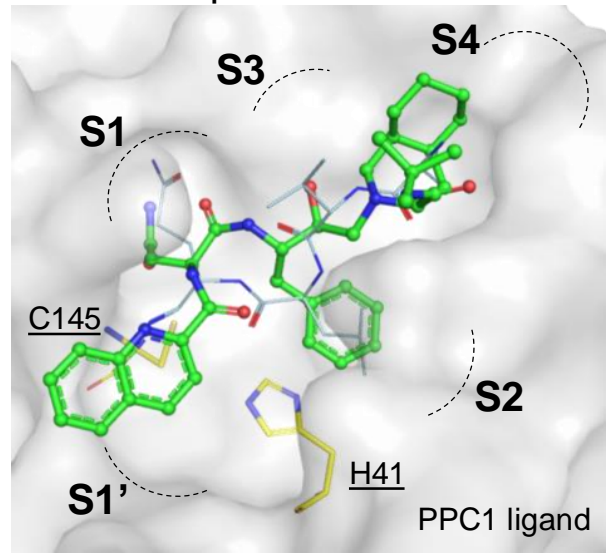

Telaprevir in PPC1

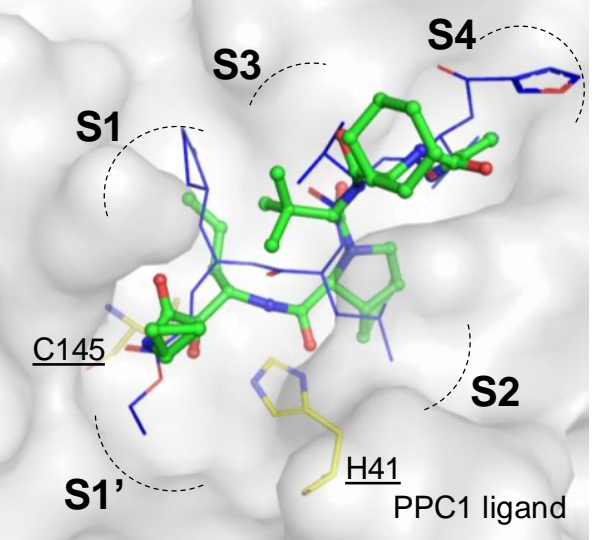

Nelfinavir in PPC2

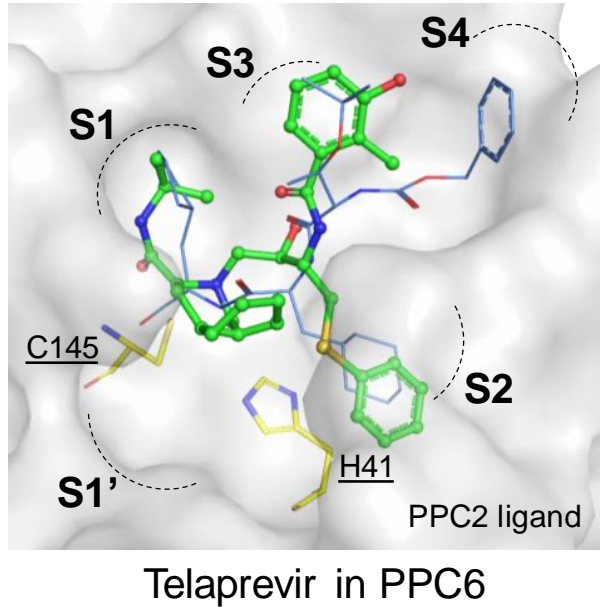

S4

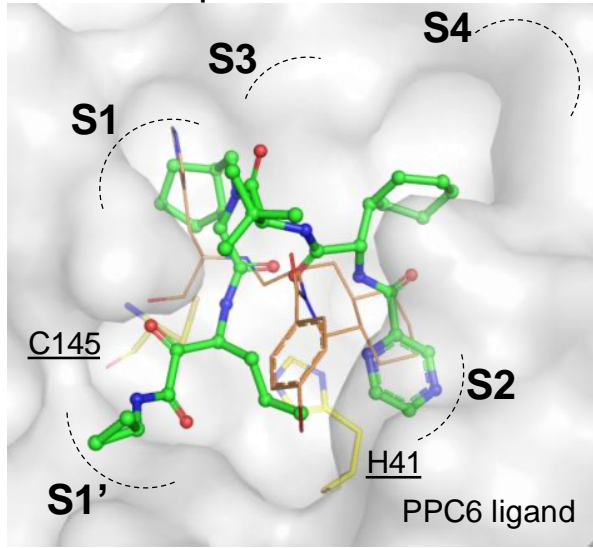

PPC1 ligand

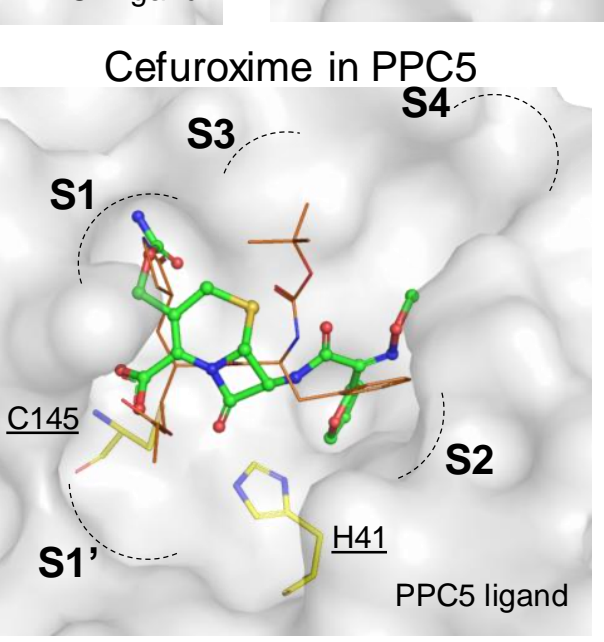

Figure S13. Drug candidate binding poses in their respective PPCs. 


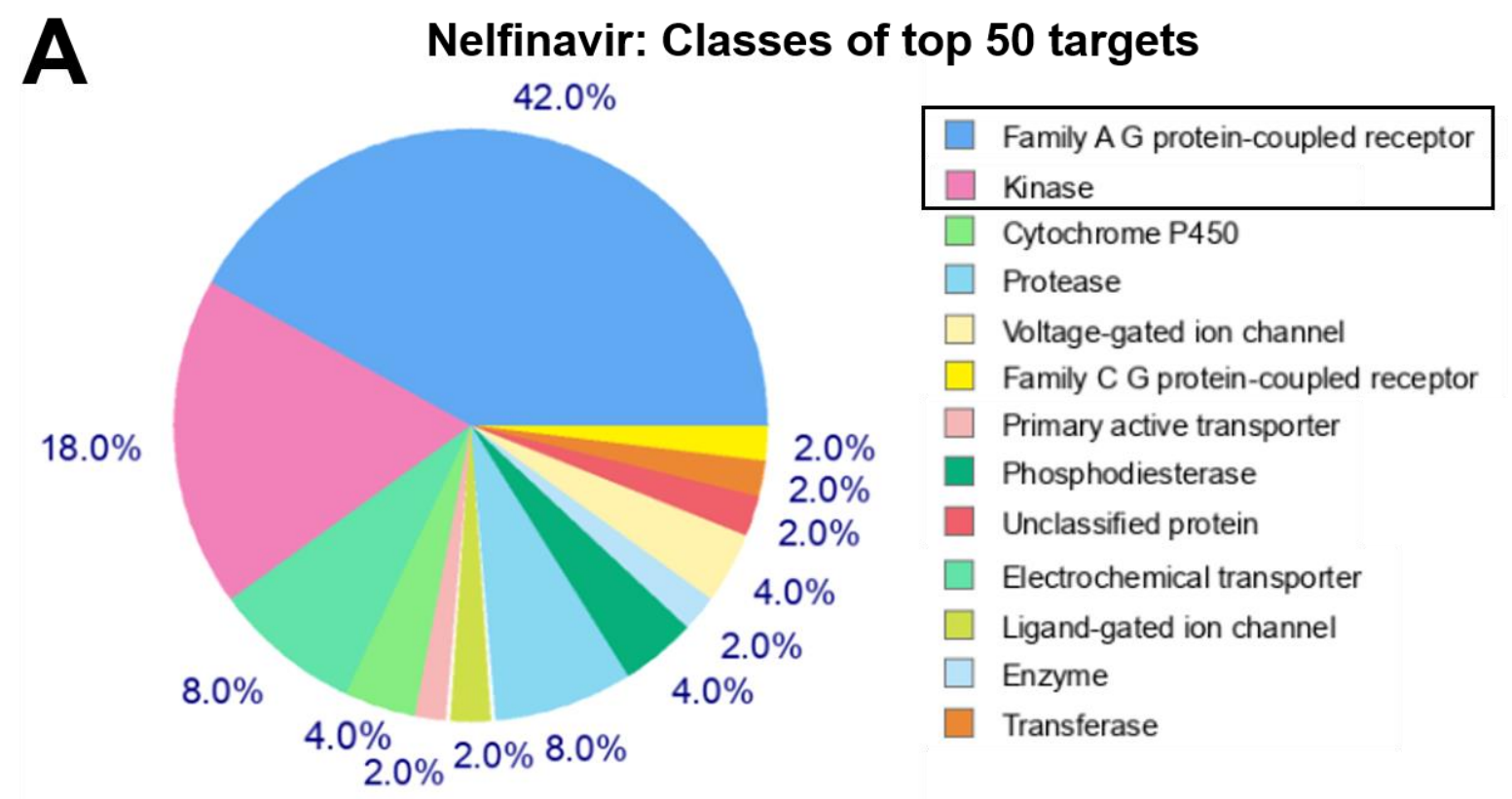

\begin{tabular}{|l|c|c|c|c|c|}
\hline \multicolumn{1}{|c|}{ Top targets from the top 2 classes } & $\begin{array}{c}\text { Common } \\
\text { name }\end{array}$ & $\begin{array}{c}\text { Uniprot } \\
\text { ID }\end{array}$ & ChEMBL ID & Target Class & Probability* \\
\hline Neurokinin 2 receptor & TACR2 & P21452 & CHEMBL2327 & $\begin{array}{l}\text { Family A p protein- } \\
\text { coupled receptor }\end{array}$ & \begin{tabular}{|l|l|l|}
\hline \\
\hline Tyrosine-protein kinase FYN
\end{tabular} \\
\hline
\end{tabular}

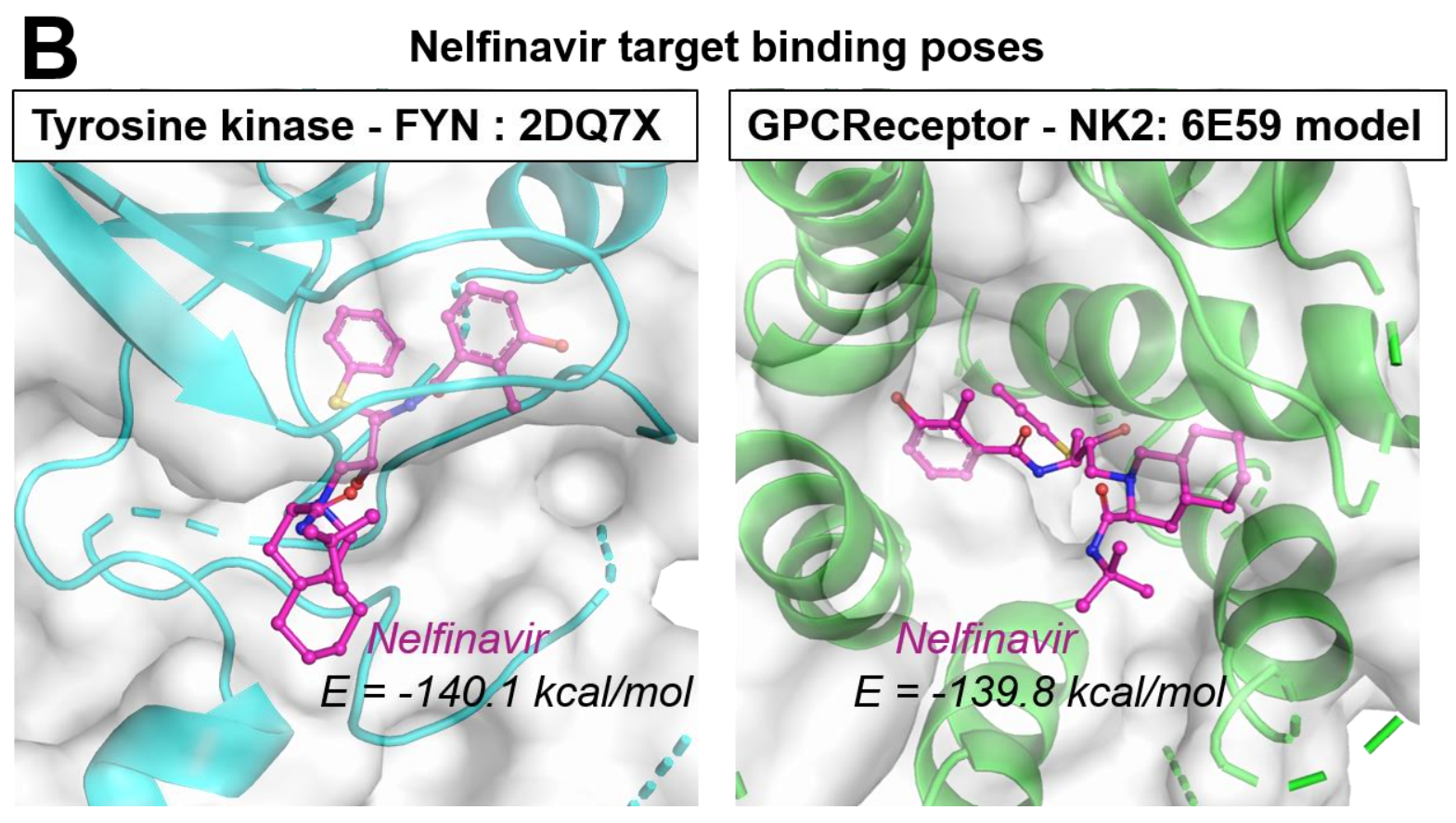

Figure S14. Nelfinavir - Predicted drug targets. (A) Top 50 predicted target classes, with major targets belonging to top classes, GPCR (Family A) and kinases. (B) The binding poses of Nelfinavir in the active sites of the top best targets, FYN kinase and Neurokinin 2 receptor are shown. 


\section{Virtual screening using unbound proteases:}

We proceed to investigate the unbound PPCs (PPC4:3E91 and PPC5: 3EA8) and unassigned unbound forms (3F9E) for evaluating their usability in screening of FDA drugs, in comparison to their bound PPC counterparts. FDA drugs were screened (protocols details in Material and Methods Section) using the unbound PPC and unassigned unbound form cavities and for their top 300 candidate poses, the drug rank correlations were calculated were compared with that of corresponding PPCS, results summarized in Figure S15. The drug ranks for the top compounds screened by unbound $v s$ bound PPCs are strongly correlated in PPC4 and PPC5 with a person correlation coefficient of 0.733 and 0.73 respectively, which justifies the unbound form assignment to these specific PPCs and consistency of PPCs (unbound or bound forms) in screening drug hits (Figure S15A). We further evaluated screening of our drug candidates by the unbound PPCs (PPC4: 3E91 and PPC5: 3EA8) and by their original PPC representatives (See Figure S15C). For the unbound PPCs, the overall drug ranks show similar pattern to the ranks in the PPCs. For example, Saquinavir originally discovered as top hit from bound PPC4 3 VB6 cavity (rank 1) is also as a top candidate from unbound PPC4 3E91 (rank 5). These screening results imply that the structures in a PPC have consensus drug ranks and similar hits. For the virtual screening results using the unassigned unbound structures (3F9E), the analysis of top 300 drug ranks revealed no correlation compared to reference PPC4 and PPC5 (Figure S15B), similarly the selected drug candidates yielded inferior ranks in screening by these unassigned forms compared to both bound and unbound PPCs (Figure S15C). This points out that the structural conformation changes observed in these unassigned structures, lead to significantly different screening results from the original PPCs. Moreover, the drug candidates screened from these unassigned active sites would be unrealistic and not ideal for bioassay testing that employs a wild-type protease. 

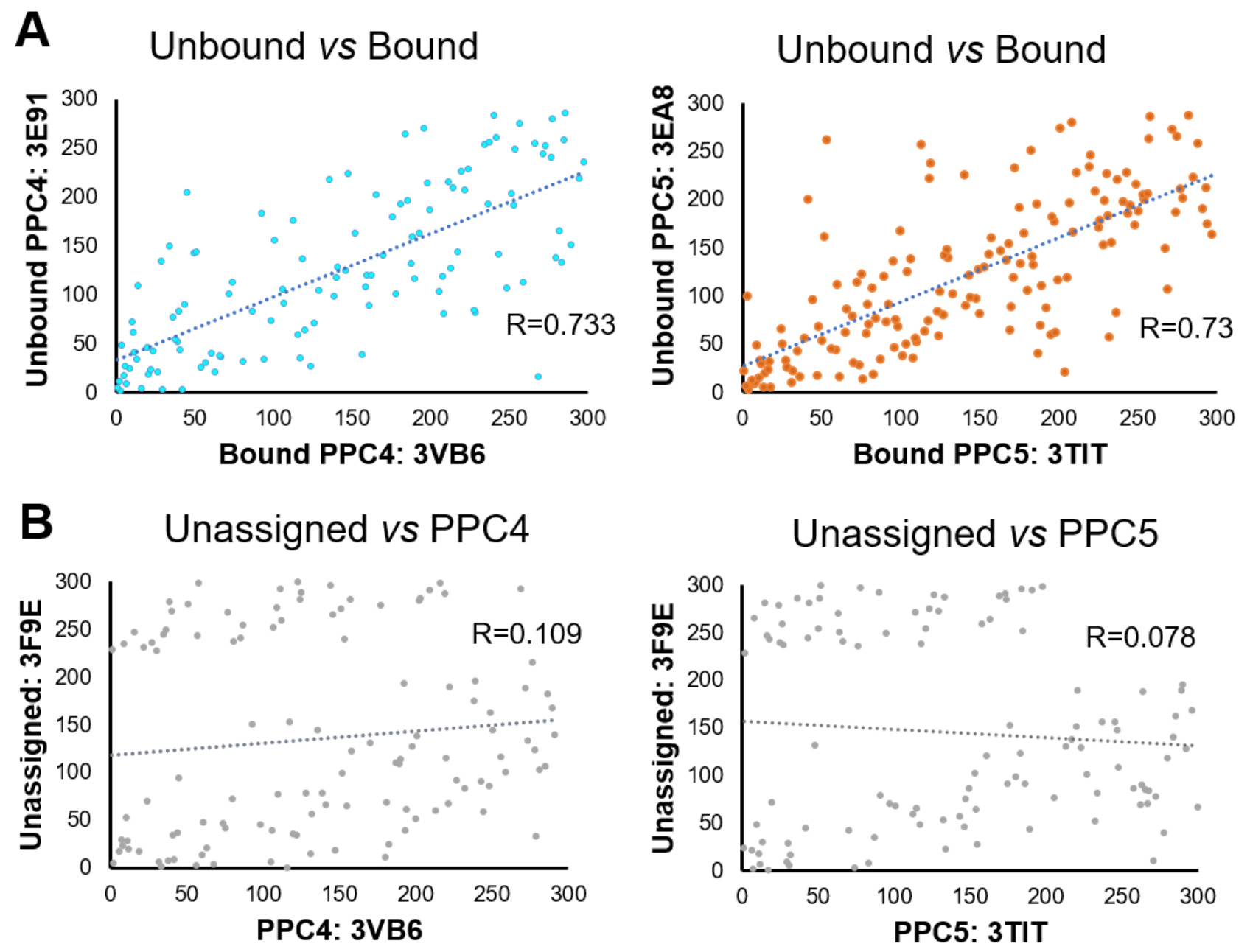

C

\begin{tabular}{cccccc} 
& PPC4 & PPC4 & PPC5 & PPC5 & Unassigned \\
\hline & $\begin{array}{c}\text { 3VB6 } \\
\text { (bound) }\end{array}$ & $\begin{array}{c}\text { 3E91 } \\
\text { (unbound) }\end{array}$ & $\begin{array}{c}\text { 3TIT } \\
\text { (bound) }\end{array}$ & $\begin{array}{c}\text { 3EA8 } \\
\text { (unbound) }\end{array}$ & $\begin{array}{c}\text { 3F9E } \\
\text { (unbound) }\end{array}$ \\
\hline Nelfinavir & 85 & 412 & 66 & 87 & 241 \\
Cefuroxime & 57 & 30 & 18 & 6 & 243 \\
Saquinavir & 1 & 5 & 2 & 7 & 229 \\
Lopinavir & 100 & 385 & 111 & 427 & 818 \\
Telaprevir & 229 & 81 & 4 & 3 & 828 \\
Boceprevir & 6 & 27 & 11 & 33 & 18 \\
Edoxaban & 29 & 134 & 28 & 26 & 869 \\
\hline
\end{tabular}

Figure S15. Virtual screening using unbound-form proteases: (A) Rank correlation of top 300 compounds screened by unbound and bound PPC cavities, (B) Rank correlation of top 300 compounds screened by unassigned vs bound PPC cavities for PPC4 and PPC5. (C) Drug candidate ranks by bound PPCs, unbound PPC and unassigned cluster virtual screening. 
(nsp5/nsp6, C-ter of 3CLpro) (nsp4/nsp5, N-ter of 3CLpro)

(nsp6/nsp7)

(nsp7/nsp8)

(nsp8/nsp9)

(nsp9/nsp10)

(nsp10/nsp11, nsp10/nsp12)

(nsp7/nsp8)

(nsp7/nsp8)

(nsp7/nsp8)

(nsp7/nsp8)
RQCSGVTFQSAVKRTIKG RQCSGVTFQGKFKKIVKG

TSITSAVLQSGFRKMAFP PCIKVATVQSKMSDVKCT PCIKYATVQSKMSDVKCT TSITSAVLQSGFRKMAFP MLDNRATLQATASEFSSL MLDNRATLQATASEFSSL RANSA VKLQNNELSPVAL RANSAVKLQNNELSPVAL SLAATVRLQAGNATEVPA SLAATVRLQAGNATEVPA DQLREPMLQSADAQSFLN DQLREPLMQSADASTFLN MYTPHTVLQANGACVLCN MYTPHTVLQANGACVLCN PRRNYATLQAENVTGLFK PRRNYATLQAENVTGLFK LWNTHTRLQS LENVAFNV LWNTHTRLQS LENVAYNV VETFYPKLQSSQAWQPGV VETFYPKLQASQAWQPGV

$\mathbf{B}$
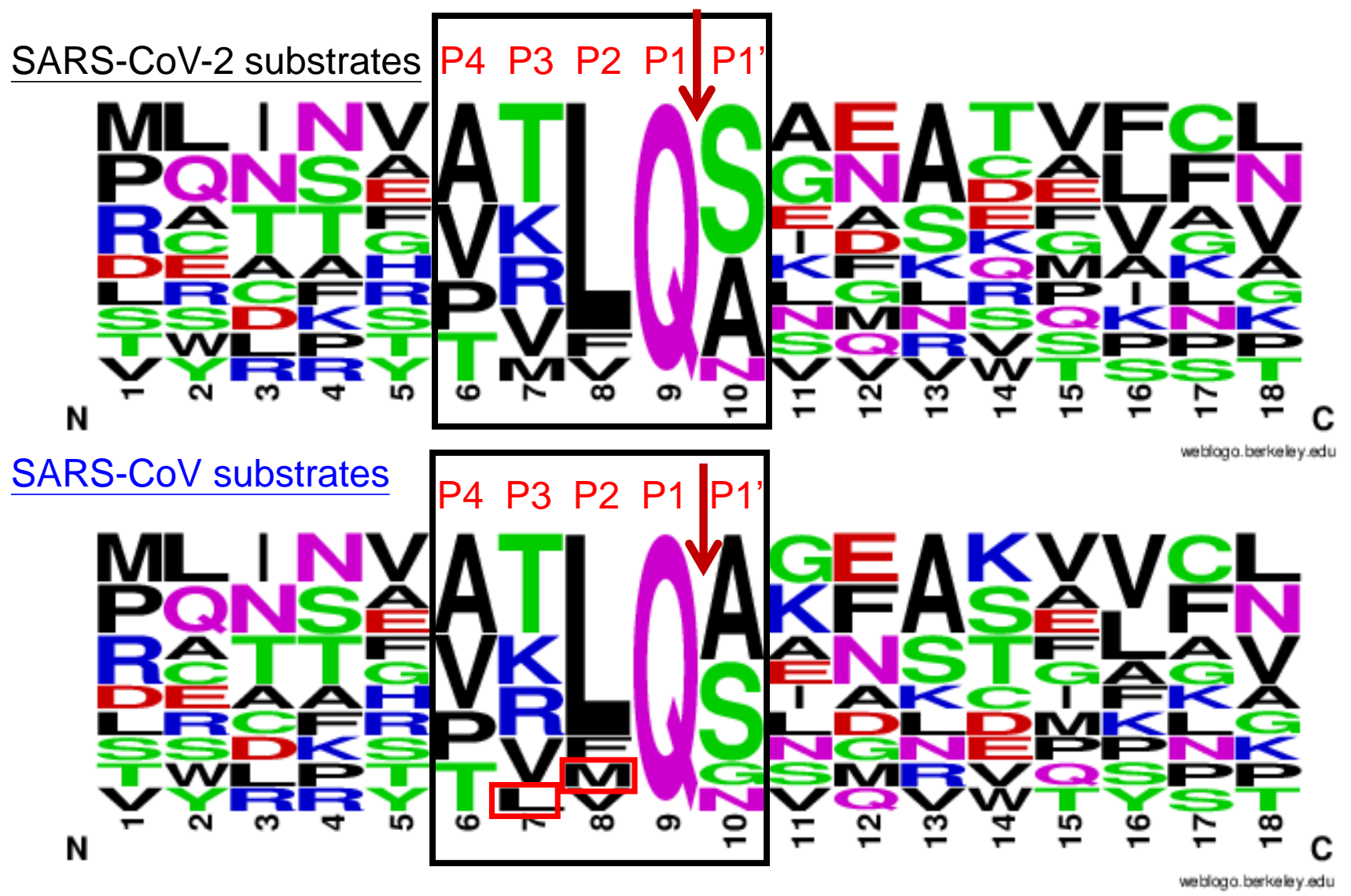

Figure S16: Substrate peptide sequences of SARS-COV and SARS-COV-2 3CL proteases. (A) protease substrate sequences and cleavage motifs in polyprotein, for SARS-CoV-2 and SARS-CoV-2, (B) Consensus substrate residues at P1'-P1 to P4 outlined in SARS-CoV-2 and SARS-CoV-2 substrate peptides, which proteolytic cleavage site (shown by yellow clipart). The uniquely different substrate residues in SARS-CoV are underlined green, of which unique residues at P1'-P1 to P4 outlined red. 


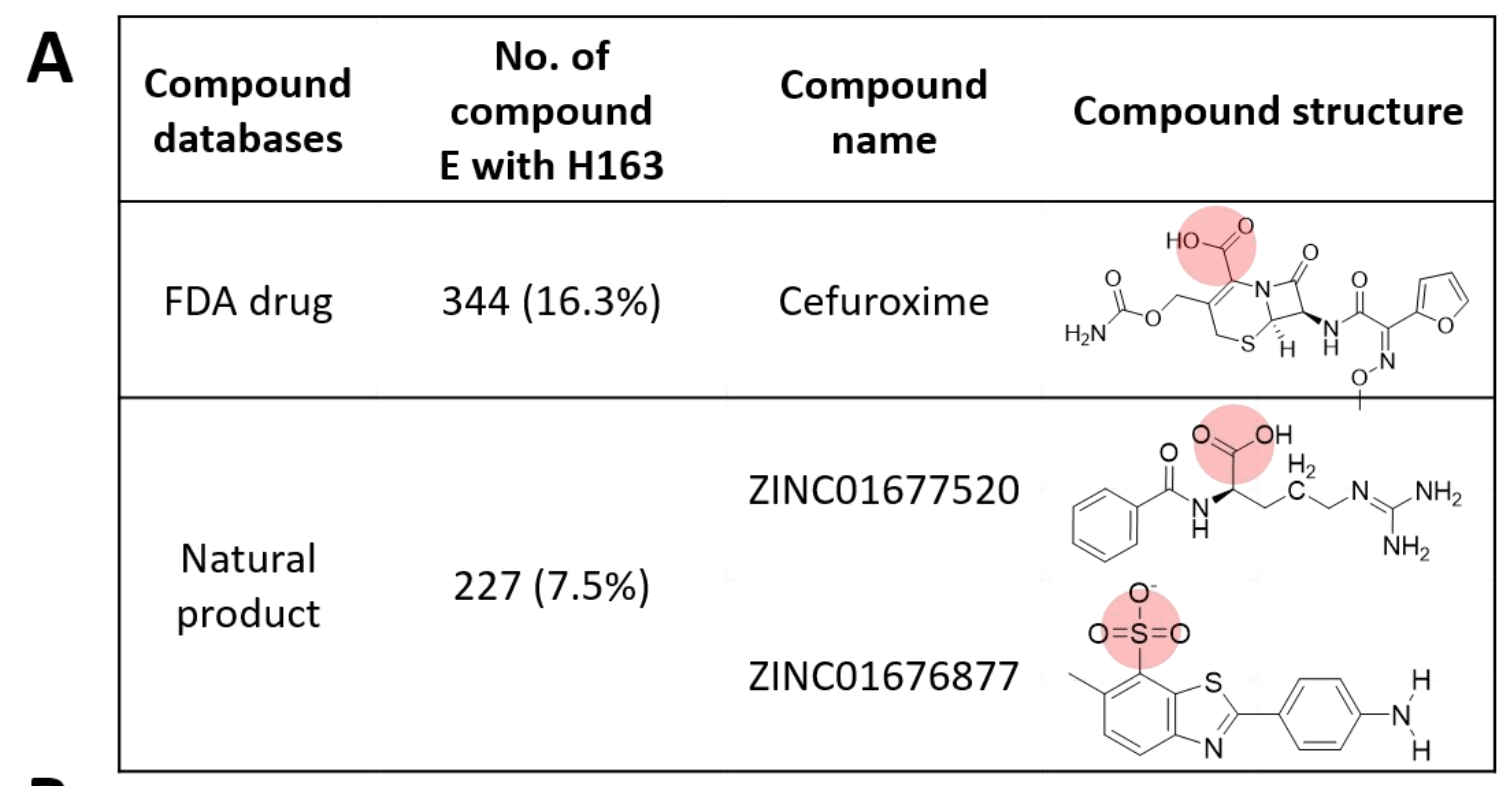

B

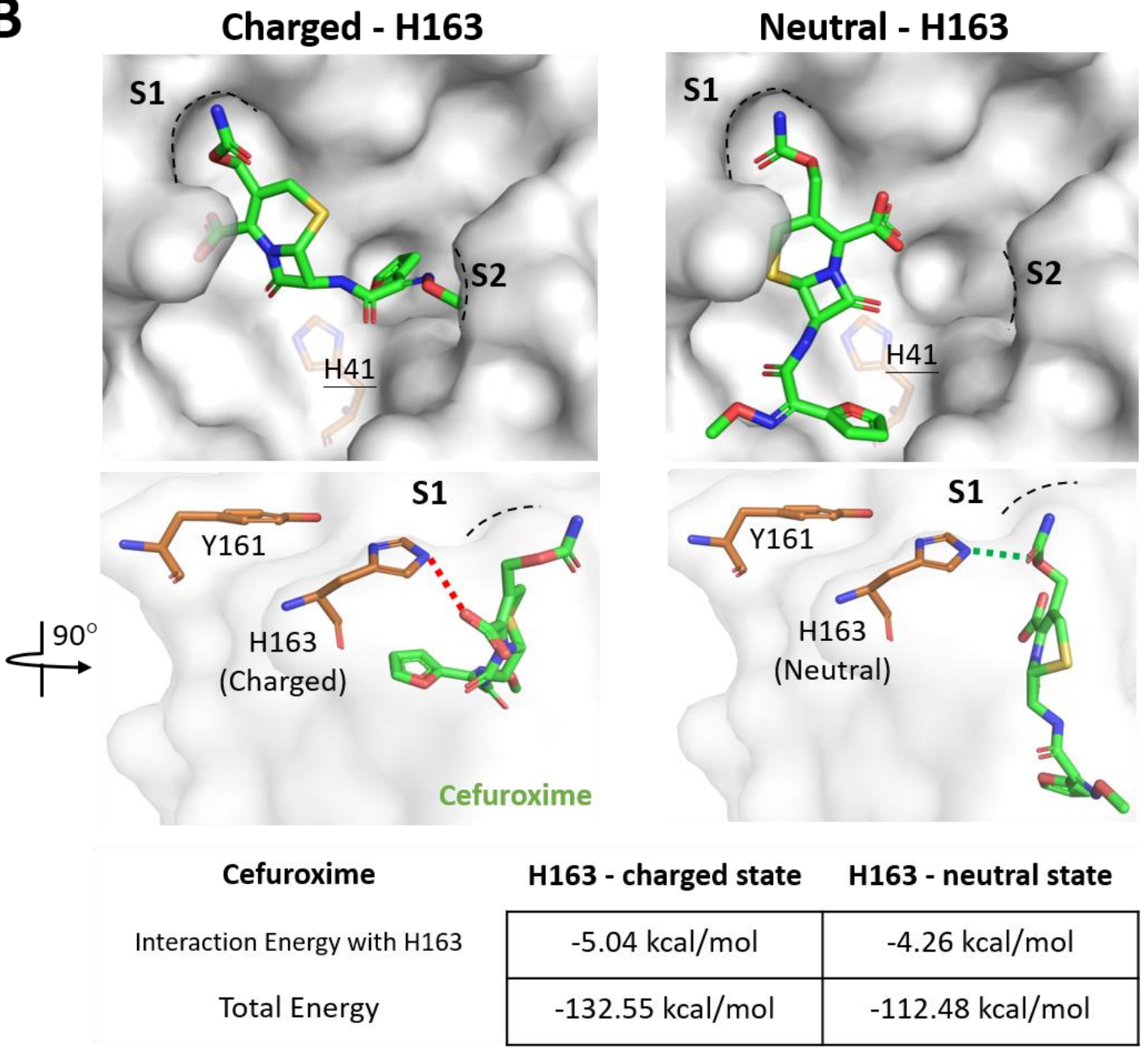

Figure S17: Screening results and interactions involving anchor residue H163. (A) Examples of compounds screened by H163-charged state screening, from FDA drugs and Natural product datasets, with the $\%$ of compounds with charge moieties (highlight red) that form E- electrostatic force (E) with H163. (B) Screening with H163 in charge and neutral states, showing Cefuroxime poses (green) in PPC5: 3TIT cavity subpockets. $90^{\circ}$ view shows the $\mathrm{H} 163$ interactions with compound moieties. The H163 interaction energy and the total pose energy for both results are shown. 


\section{REFERENCES}

1. Achilonu, I.; Iwuchukwu, E. A.; Achilonu, O. J.; Fernandes, M. A.; Sayed, Y., Targeting the SARS-CoV-2 Main Protease Using FDA-Approved Isavuconazonium, a P2-P3 Alpha-Ketoamide Derivative and Pentagastrin: An In-Silico Drug Discovery Approach. J Mol Graph Model 2020, 101, 107730. 